\title{
High gradient linac for proton therapy
}

\author{
S. Benedetti, A. Grudiev, and A. Latina
}

CERN, CH-1211 Geneva-23, Switzerland

(Received 23 January 2017; published 13 April 2017)

\begin{abstract}
Proposed for the first time almost 30 years ago, the research on radio frequency linacs for hadron therapy experienced a sparkling interest in the past decade. The different projects found a common ground on a relatively high rf operating frequency of $3 \mathrm{GHz}$, taking advantage of the availability of affordable and reliable commercial klystrons at this frequency. This article presents for the first time the design of a proton therapy linac, called TULIP all-linac, from the source up to $230 \mathrm{MeV}$. In the first part, we will review the rationale of linacs for hadron therapy. We then divided this paper in two main sections: first, we will discuss the rf design of the different accelerating structures that compose TULIP; second, we will present the beam dynamics design of the different linac sections.
\end{abstract}

DOI: 10.1103/PhysRevAccelBeams.20.040101

\section{INTRODUCTION}

Hadron therapy refers to the treatment of tumors with hadrons. Though different ions, like He, are under study, the two main ones used in daily treatments are carbon ions and protons, with the latter taking the bigger part. At the time of writing, November 2016, 71 facilities are in operation in the world, ten of which can accelerate also carbon ions [1].

Cyclotrons, for protons, and synchrotrons, for carbon ions and protons, are the two accelerator types used in the above-mentioned facilities. Cyclotrons in particular proved to be a very suitable technology for proton therapy facilities, which require beam energies up to $250 \mathrm{MeV}$, and so do not encounter the ultrarelativistic limitations of cyclotron technology. Moreover, the relatively low beam rigidity permits the use of magnets of reasonable size and power consumption.

In this very competitive and fast growing market, a linac solution could seem unreasonable. Nevertheless, both cyclotrons and synchrotrons present drawbacks that linear accelerators easily overcome. Cyclotrons main drawback is represented by the fixed beam extraction energy. As a result, to target the tumor at different depths into the patient body, movable absorbers are placed in the beam transport line to passively reduce the beam energy. This causes the loss of more than $99 \%$ of the beam and the activation of the area. In addition, the beam gets scattered and secondary particles may travel towards the patient. Synchrotrons on

\footnotetext{
*Also at EPFL, Lausanne, Switzerland.

Previous address: TERA Foundation, via Puccini 11, Novara, Italy.

stefano.benedetti@cern.ch

Published by the American Physical Society under the terms of the Creative Commons Attribution 4.0 International license. Further distribution of this work must maintain attribution to the author(s) and the published article's title, journal citation, and DOI.
}

the other hand enable the tuning of the extracted beam energy actively. This takes about $1 \mathrm{~s}$, resulting in long treatment time of an average volume tumor, with repercussion on the patient comfort and the number of patients treated per day, so ultimately on the economical sustainability of the facility. So, ultimately, on the economical sustainability of the facility.

The key advantage of linacs lies in the possibility to actively change the output beam energy, as proposed by TERA Foundation and discussed in [2]. This can be accomplished by varying electronically the rf amplitude and phase in the last active accelerating structure at a repetition rate typically of 100 to $200 \mathrm{~Hz}$. These features translate into a quick treatment, with no activation and no scattering of the beam.

Linacs for proton therapy were first proposed in 1991 [3]. This solution was taken up by TERA Foundation, which in 1994-1995 designed in detail a $230 \mathrm{MeV}$ linac for proton therapy [4] and, in collaboration with CERN and INFN, first proved the feasibility of a $3 \mathrm{GHz}$ accelerating structure for protons $[5,6]$. Since 2001 the activity of TERA Foundation mostly focused on the so-called cyclinac concept. In this solution a commercial cyclotron accelerates particles up to tens of $\mathrm{MeV}$, which are then boosted by a linac up to the energies of medical interest, i.e. 70 to $230 \mathrm{MeV}$ in the case of protons.

Following the design of Ref. [4], the ENEA (Italian national agency for new technologies, energy and sustainable economic development) group of Frascati, Italy, worked on an all-linac solution, with an rf quadrupole (RFQ) and a drift tube linac (DTL) system covering the particle acceleration up to $40-70 \mathrm{MeV}$ [7], to be followed by the coupled cavity linac (CCL) designed by TERA $[5,6]$. All these activities have been described in the review paper of Ref. [2].

Arguably, one of the most recent breakthroughs in the field is represented by the $750 \mathrm{MHz}$ CERN RFQ [8]. This solution was specifically designed to inject particles at 
$5 \mathrm{MeV}$ into a $3 \mathrm{GHz}$ DTL structure as the one proposed by ENEA. Based on these developments, A.D.A.M [9], a spinoff company of CERN, is building on CERN premises a commercial all-linac machine for proton therapy, based on a RFQ-DTL complex and a CCL solution $[5,6]$.

\section{THE TULIP ALL-LINAC SOLUTION}

All the developments on linacs for proton therapy previously listed are focused on efficient acceleration and control of the beam. In recent years however, the footprint has became increasingly important, since proton therapy facilities have as a final goal the installation in hospital buildings, where dimensions are an issue. As a result, the number of centers with just one treatment room is growing [1].

TERA Foundation first proposed a single-room facility based on a cyclinac concept in 2013 [10], called TULIP (TUrning LInac for Proton therapy). The idea consists in having a commercial cyclotron on the floor, which injects into a linac mounted on a rotating structure around the patient (Fig. 1).

To make this structure shorter, TERA launched a high gradient research campaign, in collaboration with the CLIC group at CERN, to investigate the high gradient limit of S-Band accelerating structures [11-13]. Based on the results of these tests, a high gradient backward traveling wave accelerating structure for $\beta=0.38$ was built and is undergoing testing $[14,15]$. This development allowed one to almost halve the length of the linac that has to be mounted on the rotating structure, saving size, weight and ultimately costs.

In the past two years, the authors started to work on a all-linac solution for TULIP, taking advantage of the development of the high frequency CERN RFQ. Hereafter, we will review in detail the first full design of TULIP all-linac.

\section{A. Comparison between cyclinac and all-linac concepts}

The cyclinac solution strength comes from the idea of accelerating up to tens of $\mathrm{MeV}$ protons in a commercial cyclotron. This has a number of advantages over linear accelerators, mainly: (i) lower complexity, being low beta

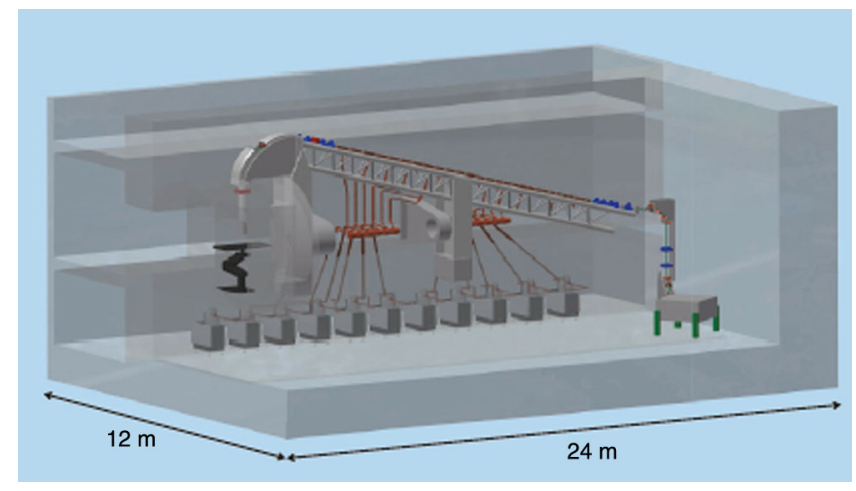

FIG. 1. Sketch of TULIP cyclinac solution (courtesy of TERA Foundation). accelerators often the most critical part of the linac chain; (ii) smaller footprint.

However, cyclotrons are not technically suited to inject particles in a linac. The transverse emittance of cyclotron beam is too large to fit into the linacs acceptance, at least the one proposed in [10]. Moreover, and most importantly, the time structure of the beam in the two machines is inherently different.

As far as the transverse emittances are concerned, the available external sources are very intense and a 25-30 MeV commercial proton cyclotron accelerates typically $500 \mu \mathrm{A}$ so that the output beam can be locally collimated to fit the transverse acceptance of the linac.

The linac longitudinal acceptance poses a more serious problem because a $3 \mathrm{GHz}$ linac with a synchronous phase of -20 , a classic value, has a phase acceptance of about $0.06 \mathrm{~ns}$ every rf pulse, which has a $0.3 \mathrm{~ns}$ period. On this very short time scale, the cyclotron beam is continuous and, as a result, $10 \%$ of the beam is accelerated and $90 \%$ is outside the longitudinal bucket of the linac.

On a larger time scale, to minimize the losses the beam injected in the cyclotron is made of $5 \mu$ s pulses either by chopping the output of the continuous electron cyclotron resonance source or, preferably, by using an intrinsically pulsed electron beam ion source [16]. The injected proton pulse is about 2 times longer than $2.5 \mu \mathrm{s}$ of the accelerated pulse. As a summary, in the longitudinal phase space, only $5 \%$ of the beam is accelerated while $95 \%$ is lost.

Another disadvantage of the cyclinac solution is that the beam dynamics, being heavily influenced by the longitudinal losses, is unstable and the beam experiences emittance growth. These aspects are discussed in more detail in Sec. VE.

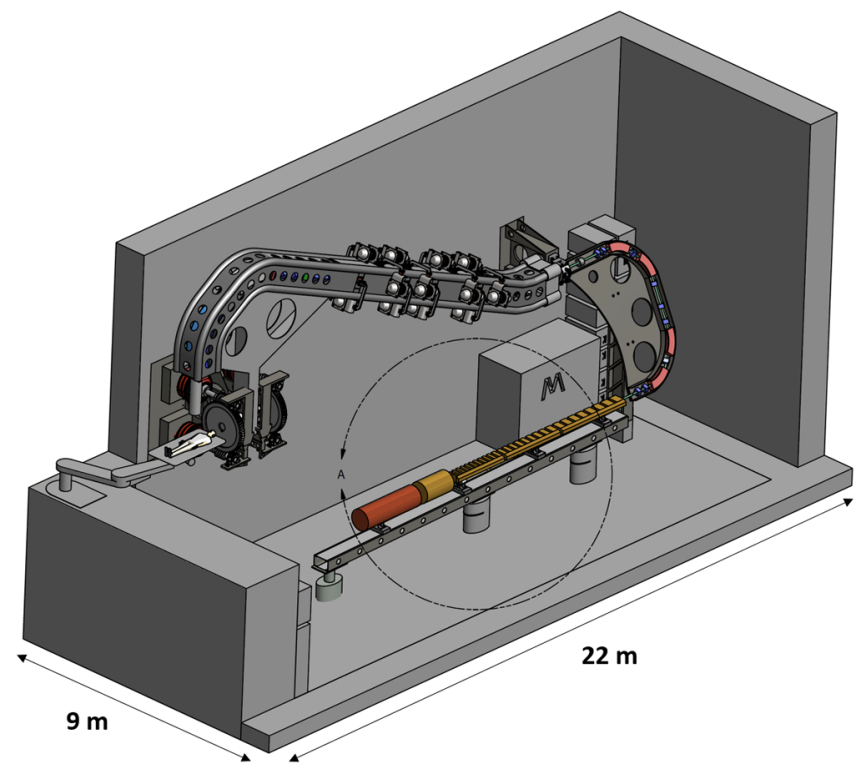

FIG. 2. Sketch of TULIP all-linac solution (courtesy of Mohammad Vaziri-TERA Foundation). 
An all-linac solution instead can reach $100 \%$ transmission with a clean beam dynamics, and thus overcome the above-mentioned issues. Linear accelerator chains are used in many laboratories around the world. However, hadron therapy linacs differ from every other application, being characterized by low current and pulsed beam. In addition, the highest possible accelerating gradient is desirable, in order to reduce the overall length of accelerators to be placed in hospital centers. These set of parameters, small aperture radius and high gradient are unique amongst linear accelerators, and thus call for a specific design. A sketch of the TULIP all-linac solution studied is presented in Fig. 2.

\section{GENERAL LAYOUT}

The design is based on a first acceleration up to $10 \mathrm{MeV}$ in $750 \mathrm{MHz}$ structures: the CERN RFQ $[8,17]$ and a newly designed interdigital $\mathrm{H}$ mode (IH) cavity. Particles are then injected into a $3 \mathrm{GHz}$ linac chain composed of a DTL, made of many side-coupled modules, up to $70 \mathrm{MeV}$, and a CCL up to $230 \mathrm{MeV}$. Table I summarizes the main parameters of the linac design.

The duty factor (DF) of the linac is currently limited by the high gradient section. A typical DF value of $0.05 \%$ would be reachable in both the RFQ, the IH and the DTL, but not in the CCL, due to the thin intracell wall thickness chosen to maximize the effective shunt impedance (ZTT). As a result, a final design should decide whether to privilege the acceleration efficiency and the linac compactness, but with a lower DF, or a higher DF but with a lower accelerating gradient or a higher peak power. The design presented hereafter will be limited by the high gradient section to a $0.01 \%$ DF, as discussed in Sec. V D 2.

The $750 \mathrm{MHz}$ RFQ was not studied by the authors, and it represents the starting point of the present work. The following three accelerating structures forming TULIP have been studied in detail from both $\mathrm{rf}$ and beam dynamics points of view. The high gradient (HG) backward traveling wave (BTW) structure was also built and tested. In the next section we start by presenting the rf design of the cavities.

\section{RF DESIGN}

Conceptually, TULIP can be split into a low gradient section, which will be placed on the ground, and a high

TABLE I. Key parameters of the all-linac TULIP solution.

\begin{tabular}{lccc}
\hline \hline Type of structure & $\begin{array}{c}\text { Output energy } \\
{[\mathrm{MeV}]}\end{array}$ & $\begin{array}{c}\text { Active } \\
\text { length }[\mathrm{m}]\end{array}$ & $\begin{array}{c}\text { Peak power } \\
{[\mathrm{MW}]}\end{array}$ \\
\hline $750 \mathrm{MHz}$ RFQ & 5 & 2 & 0.4 \\
$750 \mathrm{MHz}$ IH & 10 & 0.9 & 0.1 \\
$3 \mathrm{GHz}$ SCDTL & 70 & 4.1 & 13 \\
$3 \mathrm{GHz}$ HG BTW & $70-230$ & 4.4 & 108 \\
\hline \hline
\end{tabular}

gradient section that will be mounted on a rotating structure, called gantry. The footprint of the facility is driven by the rotating structure, which has to allocate, together with the high gradient linac, also the high energy beam transfer line (HEBT) and the beam diagnostic. As a result, about ten meters are available on the ground to install the linacs that have to boost the particles up to $70 \mathrm{MeV}$. This length has been fully exploited, in order to minimize the power consumption for a given energy gain:

$$
\Delta W=\sqrt{Z T T \cdot P \cdot L},
$$

where ZTT is the effective shunt impedance of the linac, $\mathrm{P}$ is the dissipated peak power and $\mathrm{L}$ is the linac active length.

In the following, the different accelerating structures will be revised. Particular attention will be given to the $750 \mathrm{MHz} \mathrm{IH}$ and to the $3 \mathrm{GHz}$ HG BTW structures, which were studied by the authors.

\section{A. The low beta section accelerators}

With the $750 \mathrm{MHz}$ RFQ as a starting point, the authors investigated the best solution to be placed afterwards, in the 5 to $70 \mathrm{MeV} / \mathrm{u}$ range. The rf design of this section was mostly driven by the optimization of the ZTT, together with machinability and thermal constraints. Breakdown (BD) limitations are not an issue here, since as previously discussed the accelerating gradient of this section is relatively low.

Different types of cavities, both TE and TM modes, were considered, at two operating frequencies, $750 \mathrm{MHz}$ and $3 \mathrm{GHz}$. A simplified geometry was considered, with constant drift tube thickness and stems radius independently on the geometric $\beta$. All the structures were studied by optimizing the cell gap at different geometric $\beta \mathrm{s}$, from 5 to $70 \mathrm{MeV} / \mathrm{u}$. The bore aperture radius chosen was $2.5 \mathrm{~mm}$, from preliminary beam dynamics considerations. The result of this study is shown in Fig. 3. The very high values of

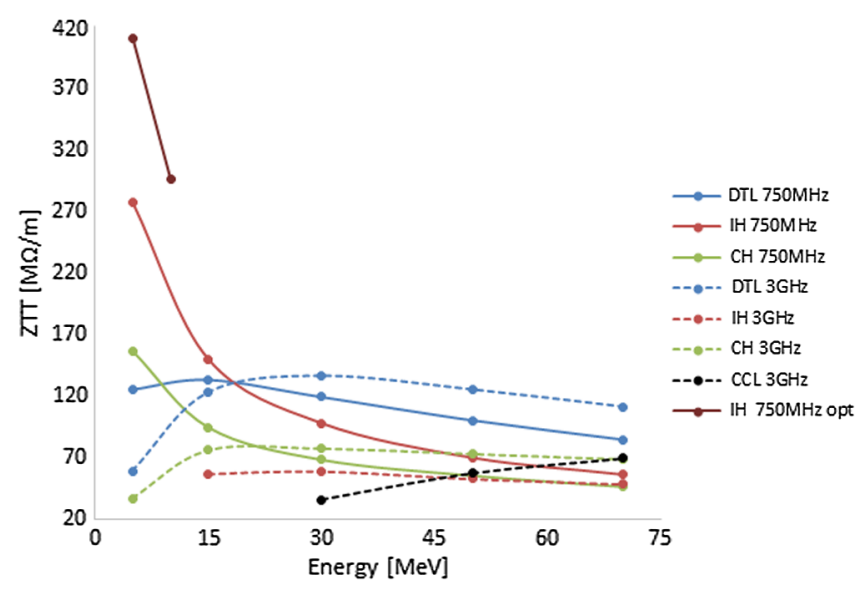

FIG. 3. ZTT as a function of the geometric $\beta$ s for the cavities considered. 


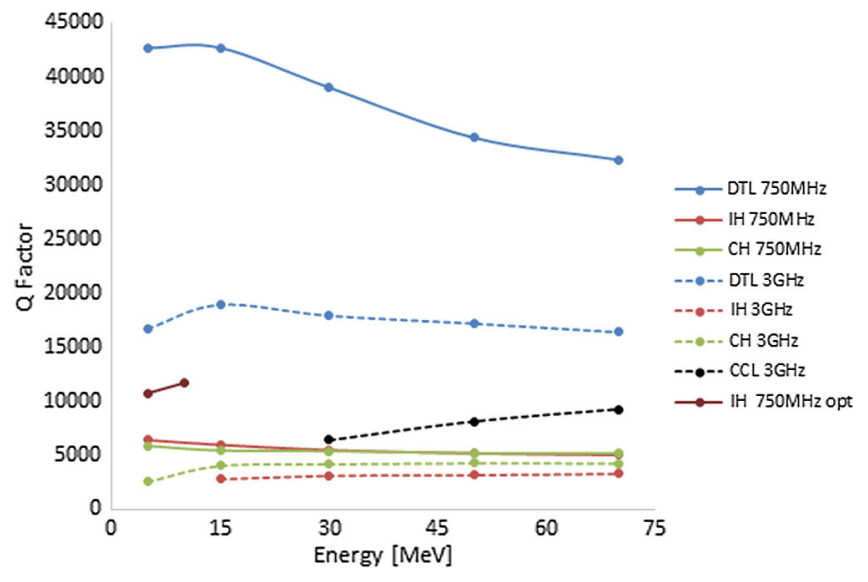

FIG. 4. Q-factor as a function of the geometric $\beta$ s for the cavities considered.

ZTT found are not common in literature, but should not surprise. Indeed, the very small bore aperture, together with the high operating frequencies, represent a uniqum amongst linac designs, and are the reason of these results.

The results of Fig. 3 can be further understood by looking at the quality factor (Q) values, presented in Fig. 4. One can notice that TM mode cavities have a significantly higher Q value. However, they pay a quite high price in terms of concentration of electric field in the nose region and transit-time (TT) factor, being $2 \pi$ mode cavities. This results in a lower overall efficiency (Fig. 5). This difference gets narrower for higher geometric $\beta \mathrm{s}$, where the TE cavities lose their advantage.

\section{1. rf optimization of TM and TE mode DTL cavities}

In TM mode DTL cavities no current flows through the stems. These have only a structural and heat dissipation purpose. Concerning ZTT, the thinner the drift tube and the drift stems, the higher is this parameter. Indeed, if it were possible to build a structure with drift tubes suspended in

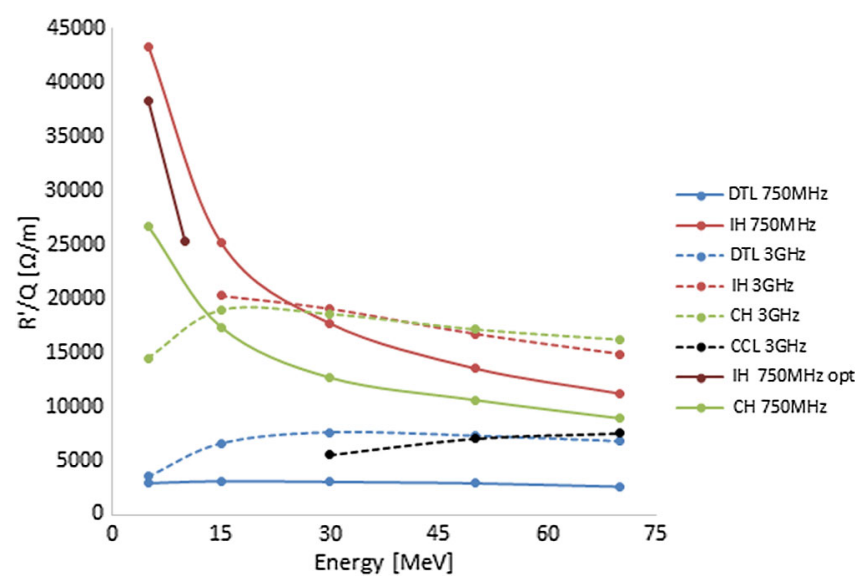

FIG. 5. R'/Q as a function of the geometric $\beta$ s for the cavities considered. the void, this would be beneficial in terms of ZTT. TM mode cavities are constant gradient structures. In the assumption of constant transit time factor, the voltage gain grows with the geometric $\beta$ of the structures, given the increased cell length. Low $\beta$ TM mode cavities usually works in $2 \pi$ mode, and this mode was considered in the present study.

TE mode DTL cavities have, on the other hand, current flowing through the stems. Here the stems and the drift tubes have again a structural and heat dissipation role, but in addition they have to force the electric field to be parallel to the $\mathrm{z}$ axis in the bore aperture region. TE mode cavities usually work in either dipole magnetic mode- $T E_{110}$, being called interdigital $\mathrm{H}(\mathrm{IH})$ - or as RFQs in quadrupole magnetic mode- $T E_{210}$, being called cross-bar $\mathrm{H}(\mathrm{CH})$. The current flowing through the stems brings to Ohmic losses that can be minimized increasing the size of drift tubes and stems. However, this reduces the electric field concentration near the $\mathrm{z}$ axis. Ultimately, a detailed $\mathrm{rf}$ optimization is needed to find the optimum ZTT for a given cell length, taking into consideration machinability and thermal dissipation constraints. A more detailed discussion on the rf optimization of TE cavities can be found in [18]. TE mode cavities work in $\pi$ mode, so they are shorter than TM mode cavities for the same operating frequency and geometric $\beta$.
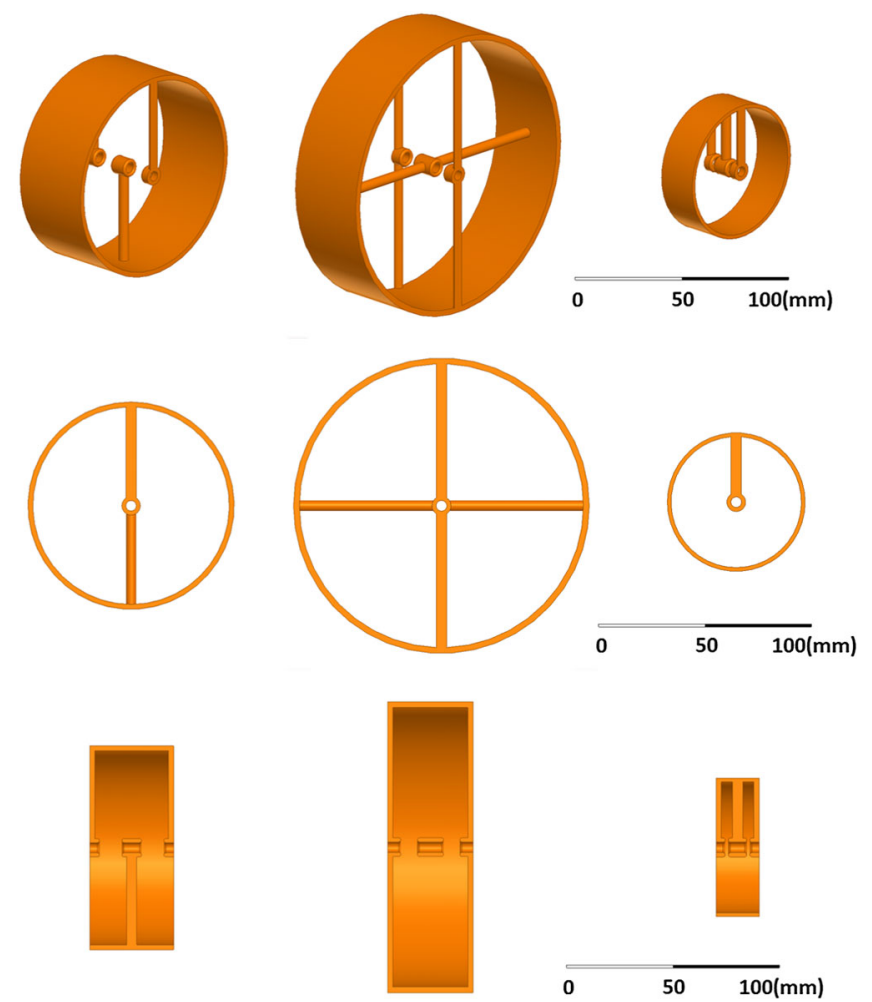

FIG. 6. Comparison between selected $5 \mathrm{MeV} / \mathrm{u}$ cells. Asymmetric view (top), transverse (middle) and longitudinal section (bottom). $750 \mathrm{MHz} \mathrm{IH}$ (left), $750 \mathrm{MHz} \mathrm{CH}$ (middle) and $3 \mathrm{GHz}$ DTL (right). 


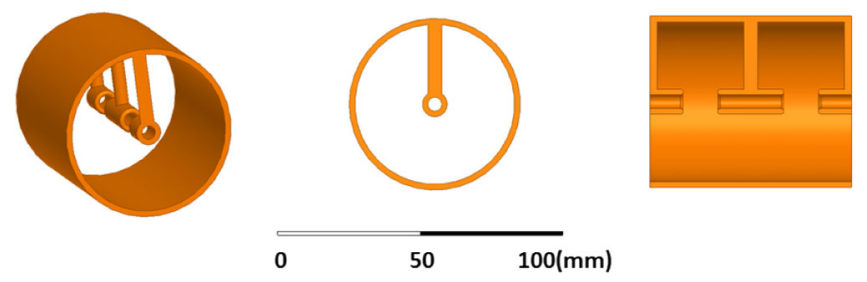

FIG. 7. $3 \mathrm{GHz}$ DTL structure at $70 \mathrm{MeV} / \mathrm{u}$. Asymmetric view (left), transverse (middle) and longitudinal section (right).
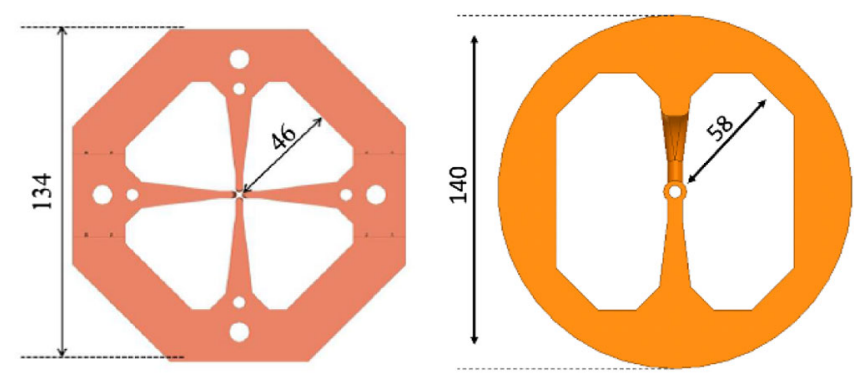

FIG. 8. Transverse section of $5 \mathrm{MeV} / \mathrm{u}$ cells. CERN high frequency RFQ (left) and IH (right). Dimensions are in $\mathrm{mm}$.

With respect to the results presented in Fig. 3, the three most promising cavities at $5 \mathrm{MeV} / \mathrm{u}$ are the $750 \mathrm{MHz} \mathrm{IH}$, the $750 \mathrm{MHz} \mathrm{CH}$ and the $3 \mathrm{GHz}$ DTL, as shown in Fig. 6. One can clearly notice the differences in terms of cell length and diameter of the three solutions.

At $70 \mathrm{MeV} / \mathrm{u}$, the $3 \mathrm{GHz}$ DTL solution reveals to be the better choice. For visual comparison, the cavity dimensions are shown in Fig. 7.

\section{B. $750 \mathrm{MHz}$ IH structure}

From the preliminary if optimization summarized in Fig. 3, it was quite clear that the $750 \mathrm{MHz} \mathrm{IH}$ cavity was the best solution in the $5-20 \mathrm{MeV} / \mathrm{u}$ regime. The simplified geometry considered in the first comparison was revised [19]. The main goal of the rf design has been to maximize the ZTT, while sticking as much as possible to the cavity geometry considered for the CERN $750 \mathrm{MHz}$ RFQ, to take advantage of the experience gained in the construction of TE cavities at this very high frequency. Indeed both RFQ and $\mathrm{H}$-mode cavities are constant voltage structures, with the only difference that a RFQ is a $T E_{210}$ bunching machine, with vanes, while a $\mathrm{H}$ mode cavity is a $T E_{110}$ accelerator, and present drift tubes between cells. This translates in overall comparable dimensions, as shown in Fig. 8.

Three energies were studied in detail: $2.5,5$ and $10 \mathrm{MeV} / \mathrm{u}$. The $2.5 \mathrm{MeV} / \mathrm{u}$ regime was studied to verify performances and feasibility of this solution in view of further developments of carbon ion projects, where an RFQ would most likely deliver up to $2.5 \mathrm{MeV} / \mathrm{u}$ particles instead of $5 \mathrm{MeV} / \mathrm{u}$. A multidimensional optimization was carried out, considering gap, drift tube thickness, stem radius, and vane distance from $\mathrm{z}$ axis. The result of this study is a remarkably improved ZTT over previously found values (see dark red curve in Fig. 3). It is interesting to notice that the improvements do not come from a higher TT factor, since the gap is unchanged, but from an increased Q value (Fig. 4).

This is the first time these values have been obtained in literature. As previously pointed out, this is due to the very small bore aperture considered, which allowed for a previously unreached high $\mathrm{rf}$ frequency. As discussed in Sec. V B, such aperture is sufficient to get full transmission of the particles bunched by the RFQ, thanks to the transverse emittances of this machine, and to the absence of space charge.

The main geometrical and accelerating parameters of the cavities studied are shown in Fig. 9, together with a view of two cells joined together.

The thermostructural analysis was performed by importing the HFSS ${ }^{\text {TM }}$ electromagnetic field distribution to the thermal and structural packages of ANSYS ${ }^{\mathrm{TM}}$. The results showed that this structure, thanks to the high ZTT and low operating gradient, does not need a cooling channel in the case of a DF up to $10^{-3}$.
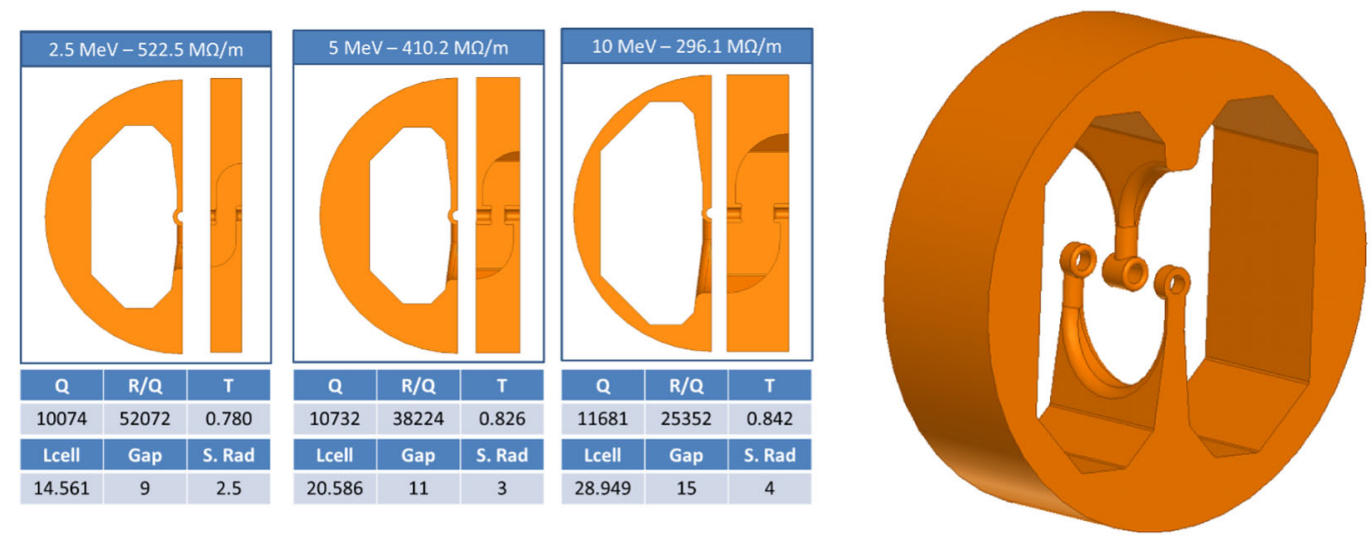

FIG. 9. Regular cell design (left) and assembly view (right) of the IH $750 \mathrm{MHz}$ cavity. 


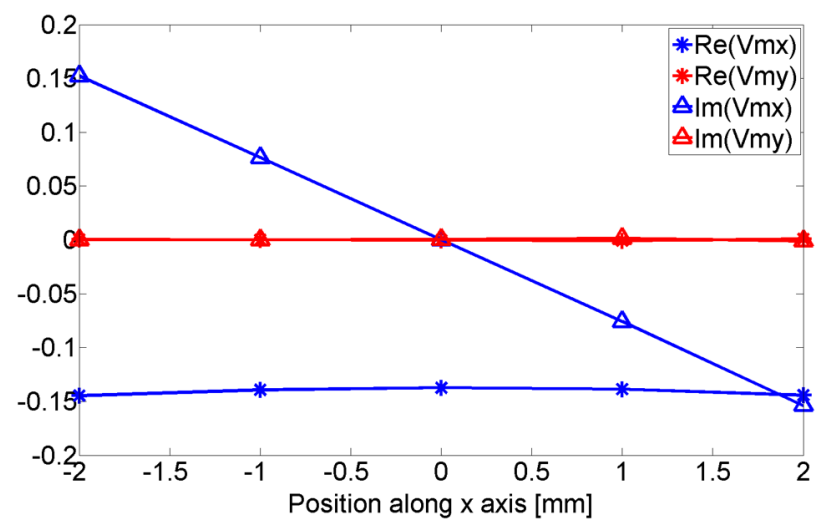

FIG. 10. Transverse voltage along the $\mathrm{x}$ axis normalized to the accelerating voltage in the $5 \mathrm{MeV} \mathrm{IH}$ cavity.

\section{Dipole kicks and transversally focusing IH cavities}

The small bore aperture, together with the thin drift tubes and large gaps of the IH cavity result in a non-negligible dipole kick. Analytically, a dipole kick is represented by a real part of transverse voltage:

$$
\vec{V}_{m}=T T \int_{0}^{L_{\text {cell }}}(\vec{E}+\vec{\beta} c \times \vec{B}) d z
$$

which is constant across the bore diameter, as shown in Fig. 10. In Eq. (2) TT is the transit-time factor. A dipole kick is typically negligible in DTL solutions that have thick drift tubes to allocate the permanent magnet quadrupoles (PMQs), and shorter gap to cell length ratios given the $2 \pi$ mode regime. In contrast, the rf defocusing, which has 90 degree rf phase shift with respect to the dipole kick component, is represented in Fig. 10 as an imaginary part of the voltage which is linear along the $\mathrm{x}$ axis, the axis on which the stems are placed.

From the results of Fig. 10 one can notice that the transverse kick is equal to about $15 \%$ of the longitudinal kick per cell, for the $5 \mathrm{MeV} / \mathrm{u}$ cell. This contribution increases with the cell length. All these effects have been taken into account in the beam dynamics studies (Sec. V B).

\section{3 GHz SCDTL}

The regular cell simplified geometry considered in Sec. IV A cannot be adopted as the reference one, due to limitations coming from structural and rf heat power dissipation considerations. A two stems geometry has been eventually considered (Fig. 11), as proposed in [7]. Such modification allows for a sufficient heat dissipation and mechanical stability, but heavily impacts on the ZTT profile presented in Fig. 3. The reduction is around 25\%.

This solution raises a slight quadrupolar asymmetry, i.e. the $\mathrm{rf}$ defocusing is stronger in the plane of the stems (y-plane in Fig. 12). The difference has been taken into account in the beam dynamics design.

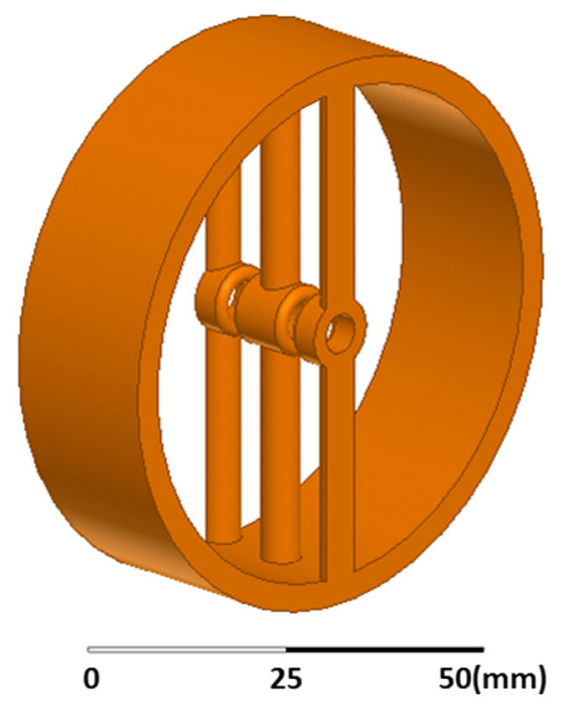

FIG. 11. The $5 \mathrm{MeV}$ reference $3 \mathrm{GHz}$ DTL cavity.

The tuning and field stabilization of a TM mode structure operating in $2 \pi$ mode at this frequency is without doubt very challenging. This issue has not been addressed yet by the authors. However, Picardi et al. presented a working solution in [7].

The advantage of such a solution comes from the possibility of using $3 \mathrm{GHz}$ klystrons, worldwide adopted
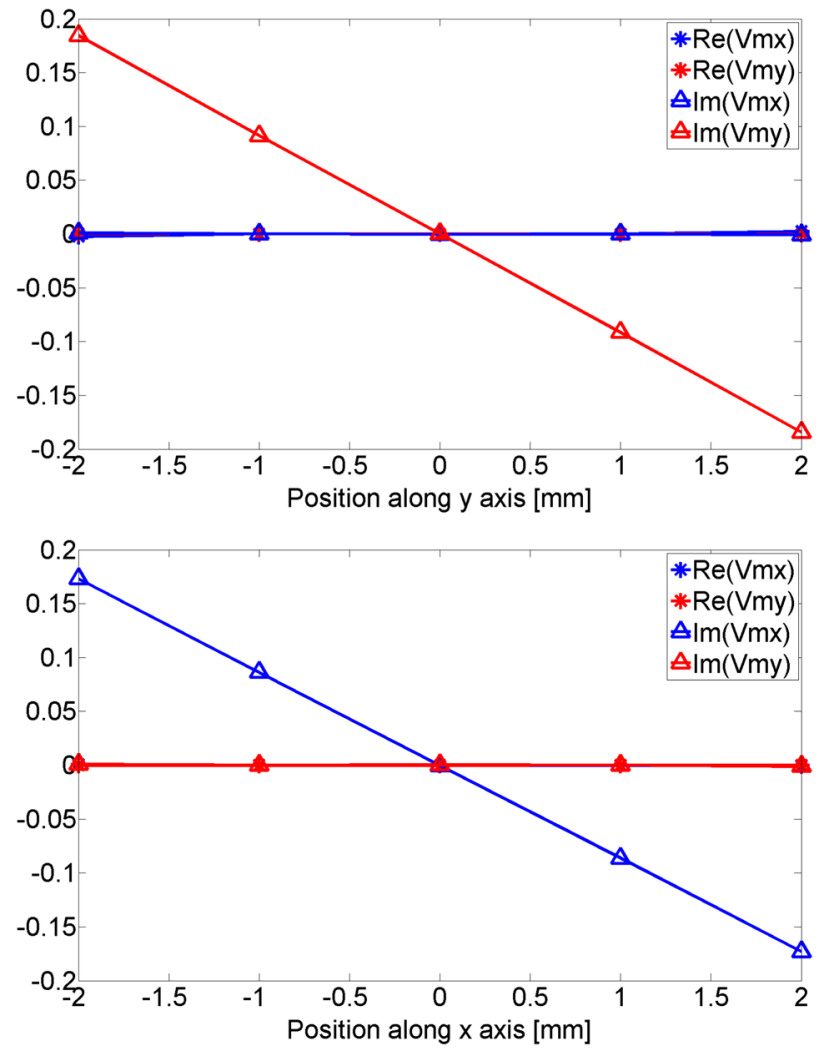

FIG. 12. Transverse voltage along the $\mathrm{x}$ and the $\mathrm{y}$ axis normalized to the accelerating voltage for the $15 \mathrm{MeV}$ DTL rf cell with symmetric stems. 
in more than 1000 electron linacs for radiotherapy, and thus very cheap rf power source.

In the present work, the rf optimization of $3 \mathrm{GHz}$ DTL cavities with the simplified geometry of Fig. 11 was performed, from 5 to $70 \mathrm{MeV}$.

\section{D. $3 \mathrm{GHz}$ high gradient BTW structure}

A high gradient backward traveling wave (BTW) accelerating structure was designed and built at CERN $[14,15]$. The main goal of the project is to define the high gradient limits of S-band cavities in terms of breakdown rate (BDR). In the design of the prototype a modified Poynting vector $\left(S_{c}\right)$ limit was used [20]. A picture of the prototype, which is $20 \mathrm{~cm}$ long and has a geometric $\beta$ of 0.38 , is shown in Fig. 13.

The BTW structure is a constant gradient magnetically coupled traveling wave cavity, with a low group velocity ranging from $0.4 \%$ and $0.2 \%$ of the speed of light. There are 12 equal length rf cells, ten regular plus two end cells. The phase advance per off cell is $\frac{5}{6} \pi$. The rf optimization was driven by the minimization of the quantity,

$$
\mu \equiv \frac{P_{w}}{E_{a}^{2}} \cdot \frac{S_{c}}{E_{a}^{2}}=\frac{v_{g}}{\omega} \cdot \frac{S_{c} / E_{a}^{2}}{R^{\prime} / Q},
$$

where $P_{w}$ is the power dissipated in one cell, $E_{a}$ is the accelerating gradient, $v_{g}$ is the group velocity, $\omega$ is the angular rf frequency, $R^{\prime}$ is the effective shunt impedance per unit length and $Q$ is the quality factor per cell. $S_{c}$ is a modified Poynting vector that has been used as a new local field to predict the breakdown behavior of the structure.

The minimization of Eq. (3) leads to the maximization of the ZTT for a given limit of $\frac{S_{c}}{E_{a}^{2}}$. The threshold was calculated by rescaling CLIC experimental data (4 MW $\left./ \mathrm{mm}^{2}, 200 \mathrm{~ns}\right)$ to pulse lengths typical of medical linacs, i.e. $2.5 \mu$ s flattop. CLIC data were rescaled according to Eq. (4):

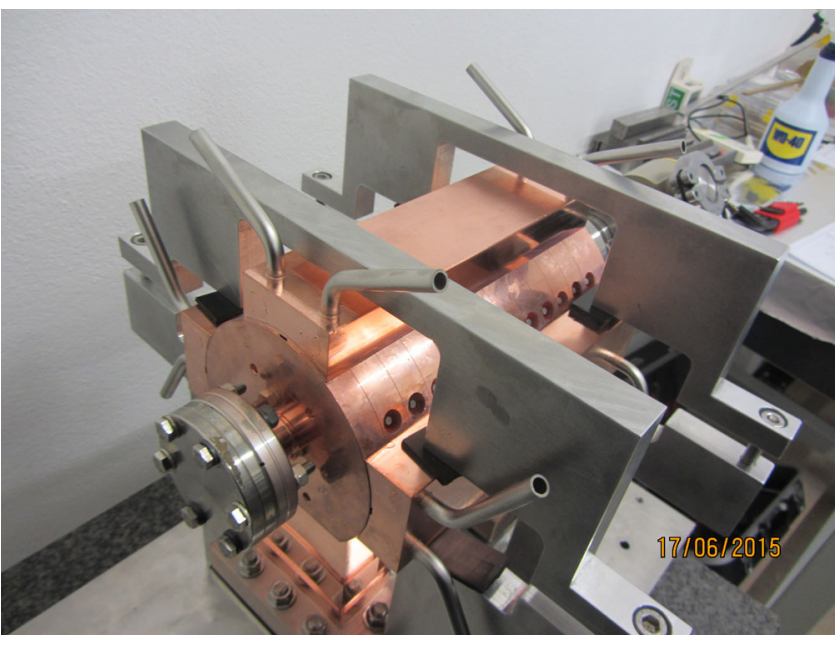

FIG. 13. The $3 \mathrm{GHz}$ BTW prototype.

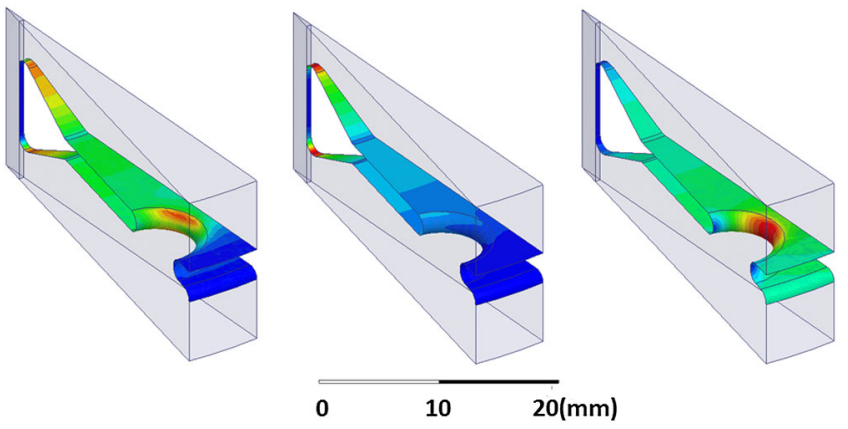

FIG. 14. Electric (left), magnetic (center) and modified Poynting vector (right) field distribution in a regular cell section $(1 / 32$ azimuthal symmetry).

$$
\frac{S_{c}^{8} \cdot t_{\text {impulse }}^{3}}{B D R}=\mathrm{const}
$$

following a research campaign on $S$-band and $C$-band single cavities carried out by TERA Foundation $[11,12,21]$. An $S_{c}$ limiting value of $1.55 \mathrm{MW} / \mathrm{mm}^{2}$ is obtained.

In this particular design, the optimum is found when $S_{c}$ is minimized simultaneously on the nose, where the electric field is maximum, and on the coupling slot, where the magnetic field is maximum, as shown in Fig. 14.

The structure was mechanically designed at CERN, as well as built, following the CLIC baseline fabrication procedure for high gradient X-band accelerating cavities. The tuning was done at CERN as well. The prototype reached the nominal phase advance between adjacent cells, and total reflection of $-60 \mathrm{~dB}$. Currently the prototype is installed in the test area under the high power test.

\section{Power recirculation in a $\mathrm{TW}$ structure}

The BTW structure designed has a power transmission ratio of about $3 \mathrm{~dB}$, i.e. half of the input power is transmitted through the structure and is coupled to either a load or a recirculating circuit. A lower $P_{\text {load }} / P_{\text {in }}$ ratio could have been obtained, but at the price of a longer filling time. To make an efficient use of TW structures, a passive waveguide component, called $3 \mathrm{~dB}$ hybrid splitter, has to be used.

A $3 \mathrm{~dB}$ hybrid is a four-port passive device. By proper sizing the geometry between the ports, it is possible to equally split the power entering from port 1 towards ports 2 and 3. In this configuration no power goes towards port 4, and there is a $90^{\circ}$ phase difference between ports 2 and 3 (Fig. 15).

In the present design, port 1 is connected to the klystron, port 2 to an rf load, ports 3 and 4 to the structure input and output cell, respectively. The power which exits from the structure, entering port 4 and being equally split between ports 2 and 3, makes interference with the power flowing from port 1 . If the phase of the rf power entering ports 1 and 4 has $90^{\circ}$ phase difference, the interference is constructive 


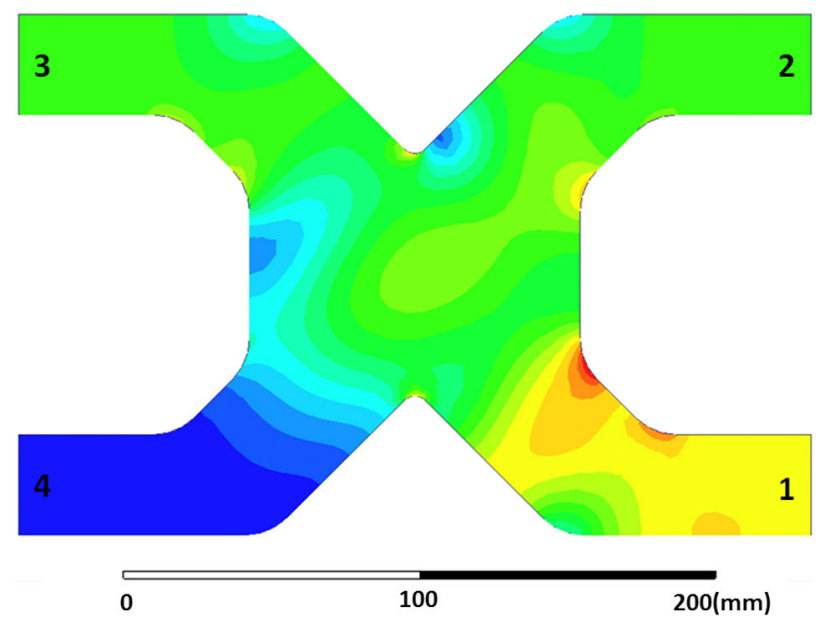

FIG. 15. $3 \mathrm{~dB}$ hybrid with power entering from port 1 .

towards port 3 , namely towards the structure, and destructive towards port 2, the load (Fig. 16).

Taking into consideration the phase difference between the accelerating structure input and output, the length of the transition waveguides which connect the $3 \mathrm{~dB}$ hybrid to the accelerating structure can be computed so that there is a $90^{\circ}$ phase difference between port 1 and port 4 . As a result, this design allows for full power recirculation into a TW accelerating structure.

The power attenuation in S-band (WR-284) waveguides is around $0.02 \mathrm{~dB} / \mathrm{m}$. As a result, the power attenuation in the $3 \mathrm{db}$ hybrid is negligible, being lower than $1 \%$. However the drawback of this solution lies in the transverse size of the assembly and in the longer filling time of a factor 4 with respect to the solution without recirculator [22]. A cut transverse view of the structure with the BTW design connected to the hybrid, and with rf power coming from port 1, is shown in Fig. 17.

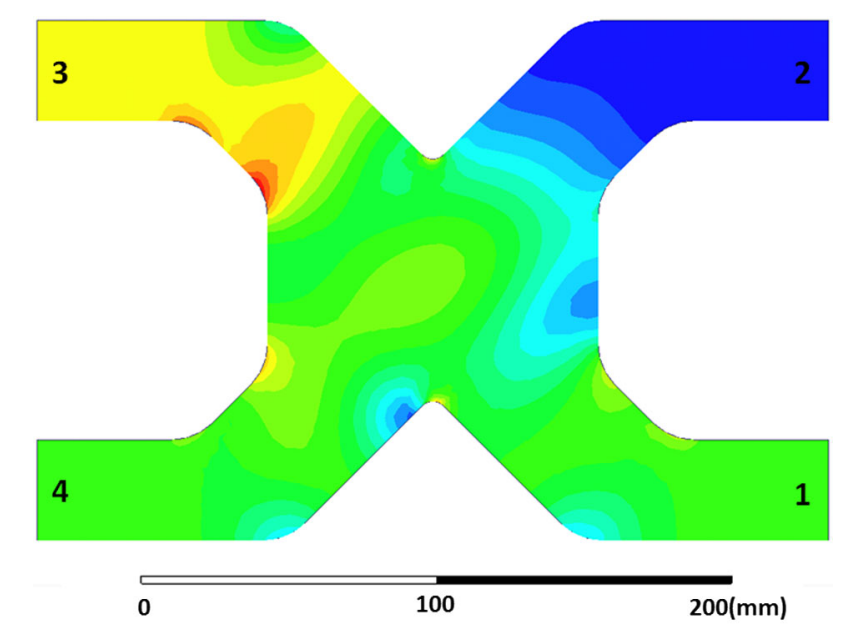

FIG. 16. $3 \mathrm{~dB}$ hybrid with power entering from port 1 and port 4 with $90^{\circ}$ phase difference.

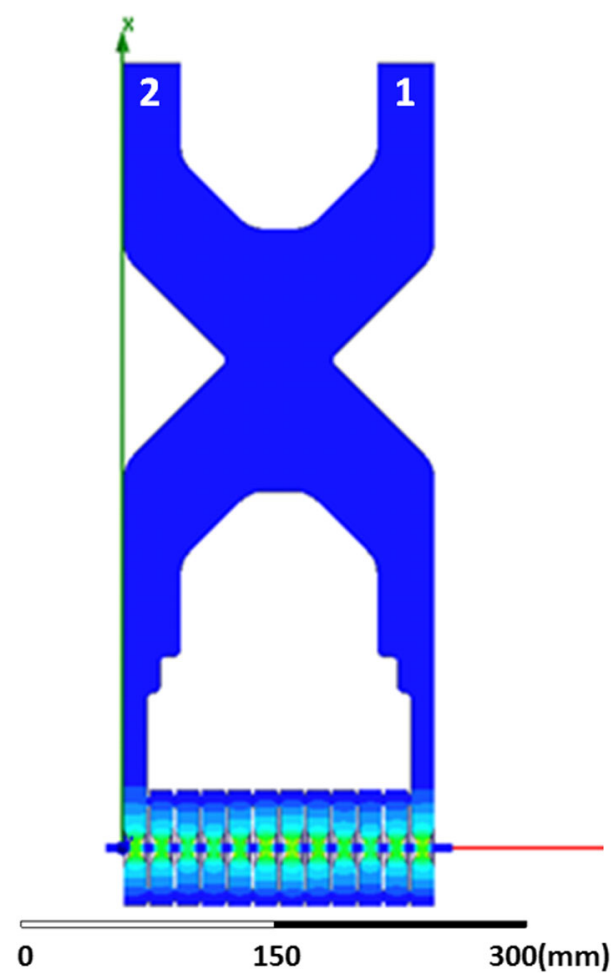

FIG. 17. Complex mag electric field distribution in BTW structure connected to the $3 \mathrm{db}$ hybrid.

\section{E. $3 \mathrm{GHz}$ high gradient CCL and comparison with the high gradient BTW}

Historically, CCLs have been a preferred solution for proton acceleration above $\beta=0.3$ or higher. However, to the authors' knowledge few works addressed the design of $3 \mathrm{GHz} \mathrm{CCL}$ above $40 \mathrm{MV} / \mathrm{m}$ accelerating gradients: one for $\beta=0.38$ [11] and a more recent study for $\beta=0.6$ [23].

The design presented in [12] has been revised, and is here presented, with a few novelties. The very same rf design methodology described in Sec. IV D has been followed, in order to compare the standing wave (SW) solution to the TW one.

The main results are summarized in Table II. The BTW and CCL designs are fully comparable in terms of maximum of the $S_{c} / E_{a}^{2}$ ratio, but also in terms of key geometrical parameters, like bore aperture and septum thickness. In the CCL case, the limit of $S_{c} / E_{a}^{2}$ is reached on the nose of the CCL, not on the coupling slot, which is a critical part but not the most critical one in this design.

The mechanical design of the CCL cavities in [5] is based on the production of two half cells that are eventually brazed together. However, a wall thickness of $2 \mathrm{~mm}$, like in the BTW solution, poses a serious challenge to the fabrication and brazing with such a method, because $1 \mathrm{~mm}$ walls would be needed. To overcome this issue, a different assembly concept has been proposed [Fig. 18 (middle)]. 
TABLE II. Main geometric and accelerating parameters of $\beta=0.38$ cells. HG BTW (left), HG CCL (center), low gradient CCL (right).

\begin{tabular}{|c|c|c|c|}
\hline Type of structure & BTW150 & SCL-HG & SCL-BL \\
\hline Phase advance per accelerating cell $[\mathrm{deg}]$ & 150 & 180 & 180 \\
\hline Wall thickness septum [mm] & 2 & 2 & 3 \\
\hline Gap $[\mathrm{mm}]$ & 7.0 & 9.0 & 5.1 \\
\hline Nose con angle [deg] & 65 & 65 & 25 \\
\hline Number of accelerating cells & 12 & 10 & 10 \\
\hline Structure active length [mm] & 189.9 & 189.9 & 189.9 \\
\hline Design accelerating gradient $\mathrm{Ea}=\mathrm{E} 0 * \mathrm{TT}$ factor $[\mathrm{MV} / \mathrm{m}]$ & 50 & 50 & 26.3 \\
\hline Max ratio surface E-field to accelerating gradient Es/Ea & 4 & 4 & 6 \\
\hline Max ratio Sc to accelerating gradient square $\mathrm{Sc} / \mathrm{Ea}^{2}[\mathrm{~A} / \mathrm{V}]$ & $2.7 \times 10^{-4}$ & $2.7 \times 10^{-4}$ & $7.8 \times 10^{-4}$ \\
\hline Quality factor $Q$ (first/last) & $6997 / 7463$ & 9136 & 8290 \\
\hline $\mathrm{R}^{\prime} / \mathrm{Q}[\Omega / \mathrm{m}]$ (first/last) & $7425 / 7369$ & 6568 & 8410 \\
\hline ZTT $[\mathrm{M} \Omega / \mathrm{m}]$ (first/last) & $52.0 / 55.0$ & 60.0 & 69.7 \\
\hline
\end{tabular}

The result of the comparison study is that the ZTT of the CCL solution, even when optimized for high gradients, i.e. $50 \mathrm{MV} / \mathrm{m}$, is higher by about $13 \%$ with respect to the BTW solution with full recirculation of the power. For comparison, the last column of the table reports the case of a CCL optimized in terms of ZTT [12]. The maximum gradient here reachable, adopting the $S_{c}$ model, is $30 \mathrm{MV} / \mathrm{m}$. It is possible to notice, graphically, the difference in the nose shape in Fig. 18. The low gradient case, called base line (BL), has a shorter gap, to maximize the TT factor, and a sharper nose, to concentrate as much as possible the $\mathrm{E}$ field near the $\mathrm{z}$ axis.

The $3 \mathrm{GHz}$ CCL structure design has a 5\% coupling factor, in agreement with a previous designed structure [16], but differs quite substantially in the coupling cell design, pieces manufacturing, and input coupler. The coupling cell design followed the goals of being easy to machine and compact in the transverse dimension.

Another novelty of this design is represented by the single coupler solution adopted. This allows individual power and phase control of each accelerating structure,

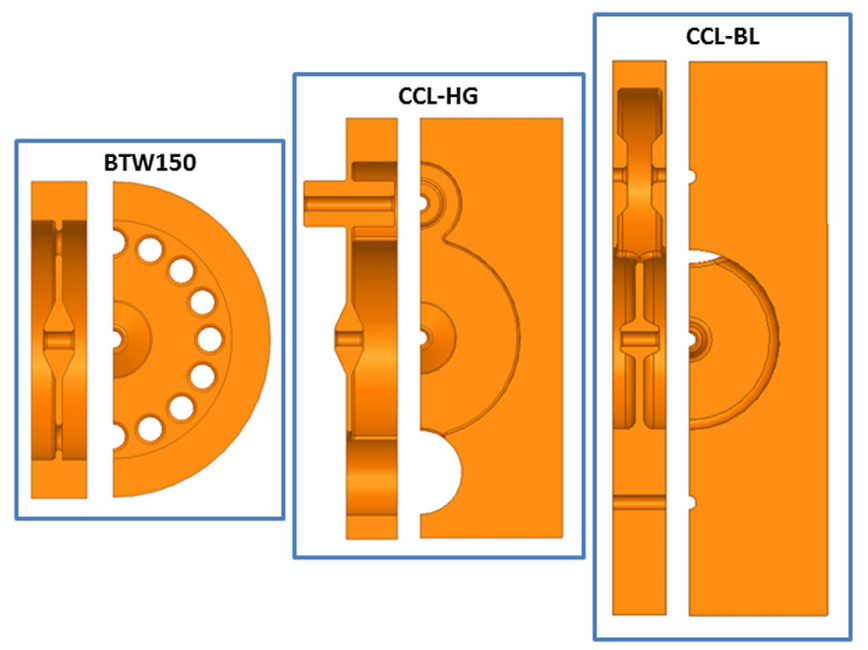

FIG. 18. Mechanical view of the optimized cells. which as discussed in Sec. VD 1 is a key feature of the TULIP project. A structure made of ten cells, $20 \mathrm{~cm}$ long, so exactly identical to the BTW prototype built, was designed and matched to the waveguides. A visual comparison of the solution is shown in Fig. 19.

The two structures were also compared in terms of $\mathrm{rf}$ thermal power dissipation. Here the CCL has two advantages: (i) a slightly higher ZTT, which translated into a
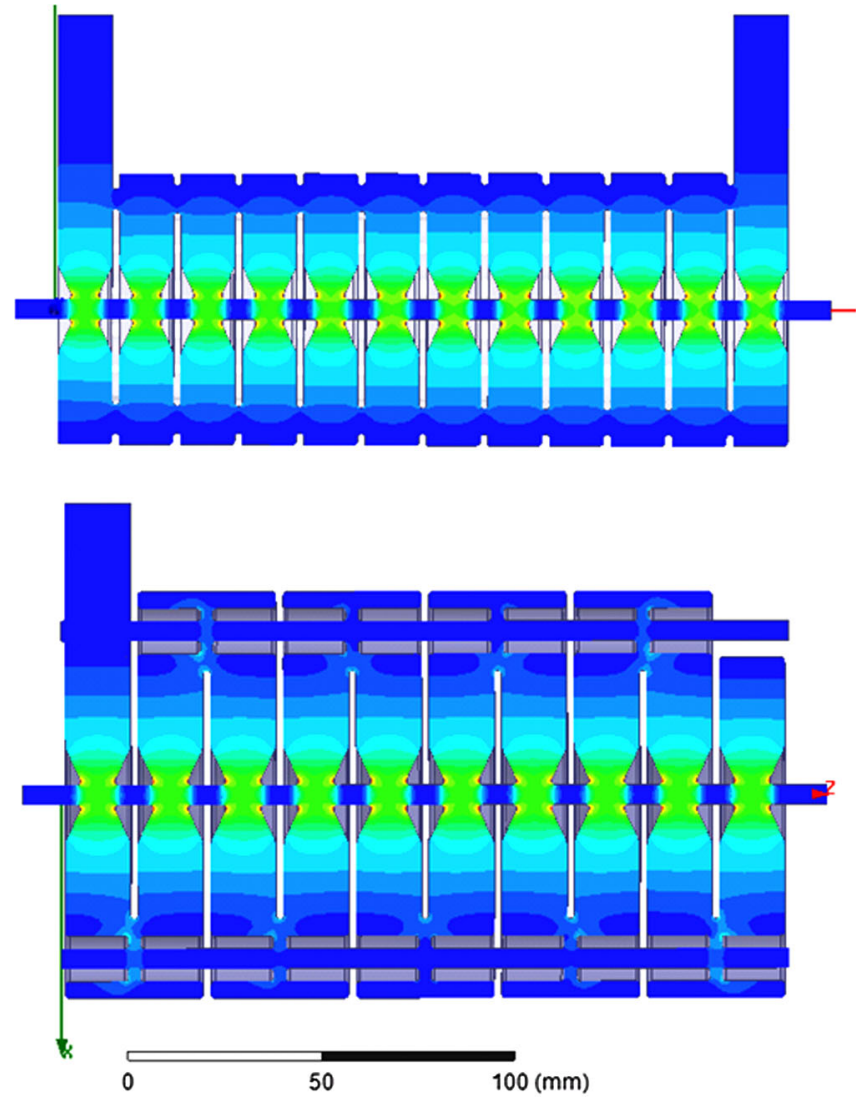

FIG. 19. Cut view with complex mag electric field distribution in the high gradient BTW (top) and CCL (bottom) structures at $\beta=0.38$. 


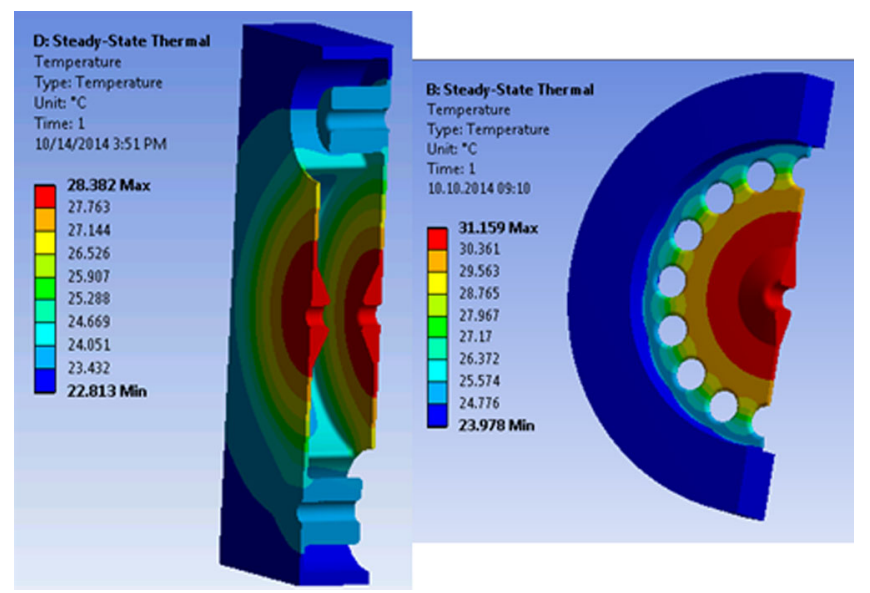

FIG. 20. Temperature distribution in the high gradient BTW (top) and CCL (bottom) structures for a $50 \mathrm{MV} / \mathrm{m}$ gradient and $0.0075 \%$ DF.

lower rf power dissipated in the copper walls for the same accelerating gradient; (ii) a higher thermal conductivity with respect to the BTW solutions, where the coupling holes are an obstacle to the power dissipation.

The temperature distributions in the two cases are shown in Fig. 20.

\section{1. rf comparison between BTW and CCL structures for a $3 \mathrm{GHz}$ high gradient linac from 70 to $230 \mathrm{MeV} / \mathrm{u}$}

The differences between a BTW and a CCL accelerating structure optimized for high gradient operations for a geometric $\beta$ of 0.38 was discussed in Sec. IVE. The

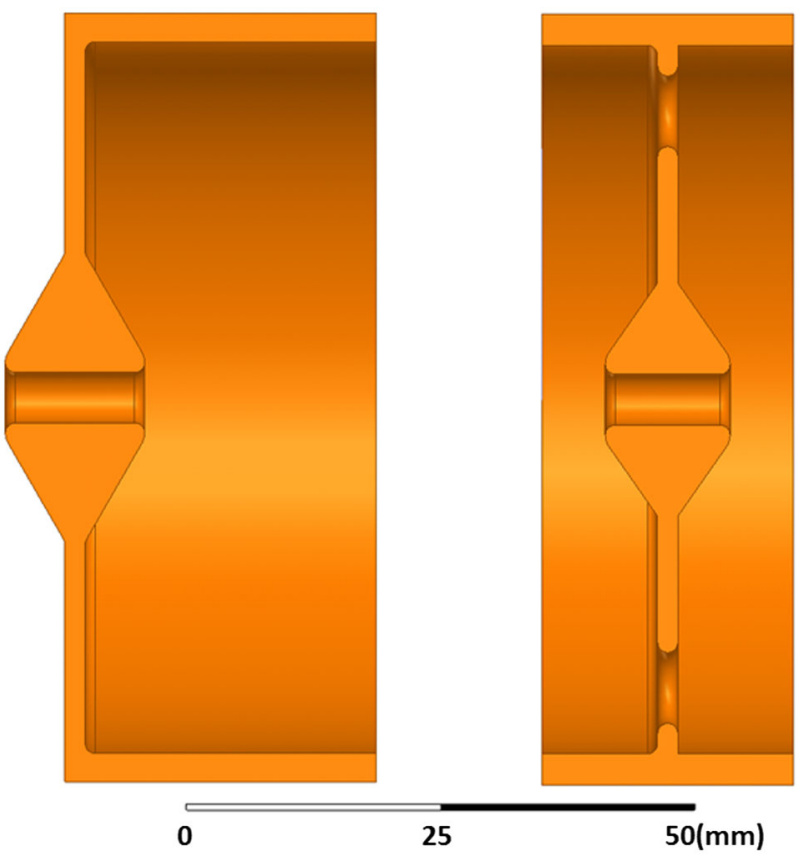

FIG. 21. Uncoupled CCL (left) and BTW (right) cells at $230 \mathrm{MeV} / \mathrm{u}$. conclusion of this comparison is an advantage of the CCL solution in terms of ZTT, and thermal power dissipation, thus maximum DF achievable. Considering the filling time, the CCL solution takes longer to reach the nominal accelerating gradient with respect to the BTW solution even if a recirculator is installed.

However, if one extends the comparison up to $230 \mathrm{MeV} / \mathrm{u}$, the results change. In particular, a $50 \mathrm{MV} / \mathrm{m}$ BTW optimized structure shows about the same ZTT as the CCL solution. The reason for this behavior comes from the difference cell length between the two solutions: the BTW, being shorter, allows for a higher optimization of TT factor (shorter gap) and nose region (sharper nose cone angle) at higher energies. The difference can be observed graphically in Fig. 21.

Three intermediate energies were studied, corresponding to 105,138 and $181 \mathrm{MeV} / \mathrm{u}$. The TT factor, Q factor and the ZTT along the linac for the two high gradient solutions are shown in Fig. 22. One can notice that the BTW can optimize better the TT factor and the nose region, but shows a lower Q factor with respect to the CCL. This difference

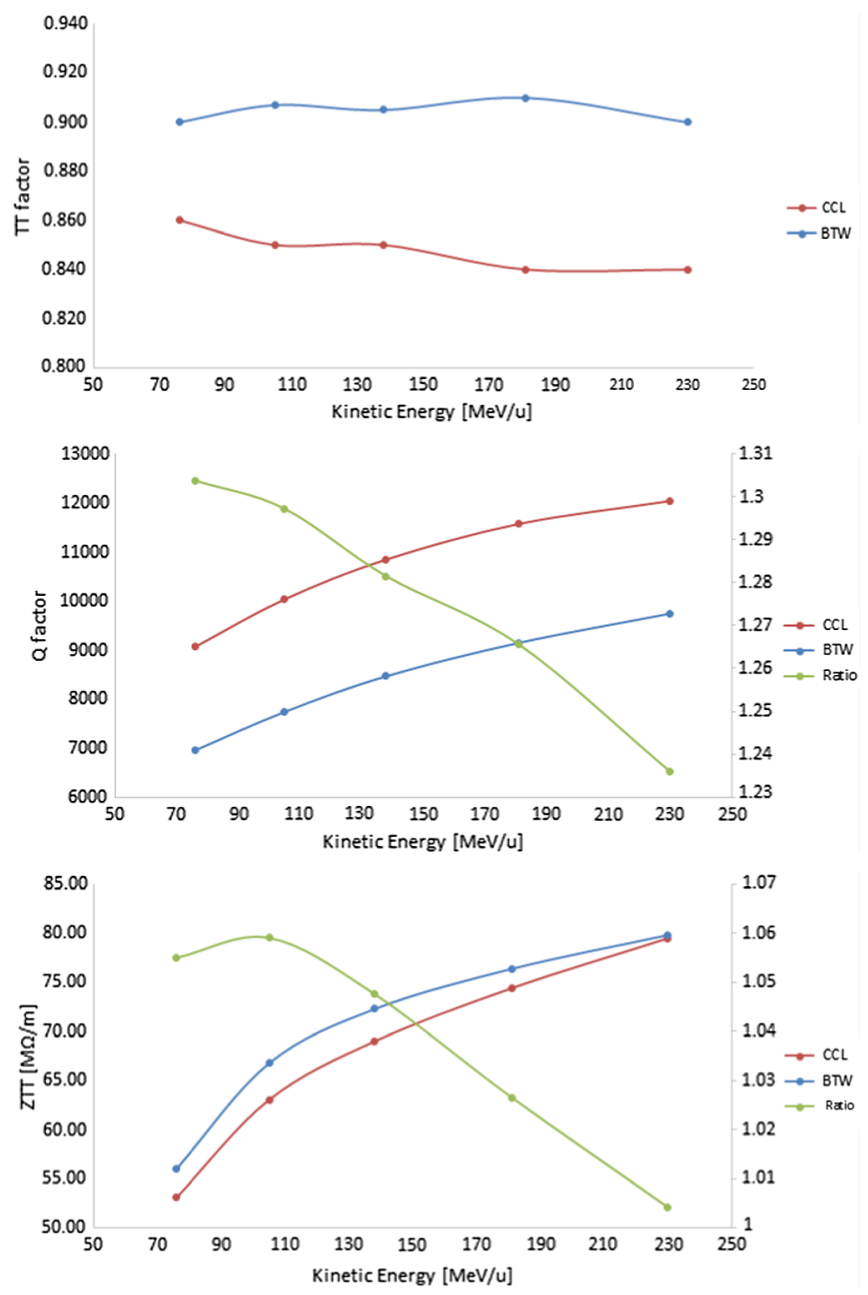

FIG. 22. TT factor (top) and ZTT (bottom) comparison between a CCL and BTW high gradient linacs. 
however gets narrower with the increase of the cell lengths, and so does the ZTT.

\section{BEAM DYNAMICS STUDY}

In this section, we review the beam dynamics design of the different linac sections.

The starting point of the beam dynamics design was the $5 \mathrm{MeV}$ output beam from the CERN $750 \mathrm{MHz}$ RFQ (Fig. 23), here simulated with the code RF-TRACK [24].

The beam emittances are relatively low, with a normalized rms transverse emittance in both planes of 0.027 pi.mm.mrad, and in the longitudinal plane of 0.015 pi.deg.MeV. These values permitted us to consider the very small bore aperture of $2.5 \mathrm{~mm}$ used in the $\mathrm{IH}$, DTL and CCL-BTW structures.

The goal of the beam dynamics design was delivering to the high energy beam transfer (HEBT) line beam between 70 and $230 \mathrm{MeV}$, with full transmission and the lowest possible normalized emittance growth. Both of these goals have been accomplished.

We will start by briefly presenting the tracking code developed for this purpose, RF-TRACK.

\section{A. A new particle tracking code: RF-TracK}

The TULIP all-linac project started with the study of the high gradient BTW linac [25]. ATW cavity complicates the particle tracking. In fact, in SW cavities the electromagnetic (EM) field oscillates according to

$$
A(x, t)=A(x) \cdot e^{-i \omega t},
$$

where $\omega$ is the angular rf frequency, and $A(x)$ is the complex magnitude spatial distribution of either the electric or the magnetic field. The electric and the magnetic field are 90 deg rotated with respect to each other.

In the TW case, a translational component of the field makes it not space independent:

$$
A(x, t)=A \cdot e^{i(k x-\omega t)},
$$
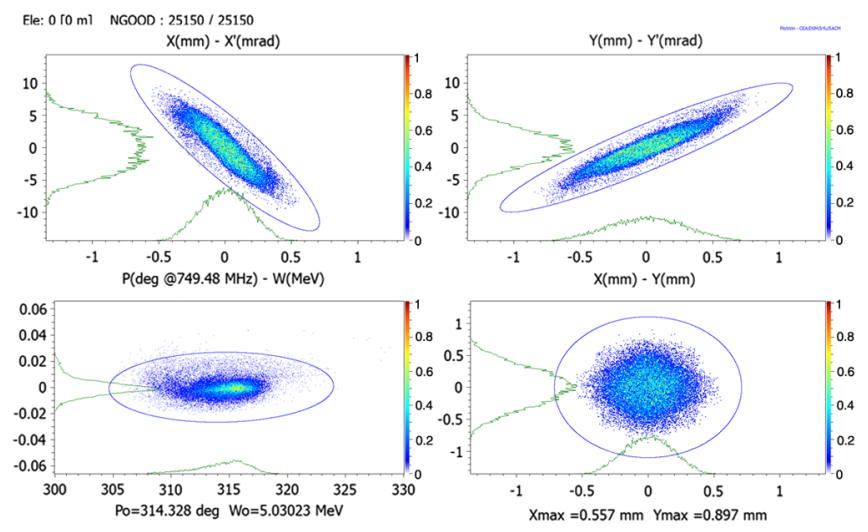

FIG. 23. Phase space of the beam at the RFQ output. where $k$ is the wave number. A negative $k x$ component characterizes a backward traveling wave.

Very few codes are capable of dealing with TW structures, since in this case both the real and the imaginary components of the field are necessary. To our knowledge, the most frequently used codes are ASTRA [26] and GPT [27]. The tracking can also be accomplished with SW codes by superimposing two SW patterns of different frequency [28]. Nevertheless, the need to work with TW field maps and to perform matchings and transmission optimizations, dynamically varying the rf input power as well as the lattice optics (see Sec. V D 1), called for development of a new tracking code: RF-TRACK. In addition, an in-house developed tool rather than a black-box tool, allowed us to add new features as necessary.

After the benchmark phase [24,25], RF-TRACK has been used to track particles start to end, i.e. the beam distribution has not been regenerated, from the RFQ output up to $230 \mathrm{MeV}$.

This approach, based on accelerating structure EM field maps, is more time consuming than an analytical tracking. In the latter, the field is generated with cylindrical symmetry from Bessel expansion of the average electric field on the $\mathrm{z}$ axis and the transit-time factor profile along the linac. However, as discussed in Sec. IV B 1 for the IH dipolar components, and in Sec. IV C for the side coupled drift tube linac (SCDTL) quadrupolar asymmetries, the linacs are not always perfectly symmetric structures. The field map approach allowed us to correctly take into consideration these aspects.

\section{B. From 5 to $10 \mathrm{MeV}$}

Protons are accelerated by the IH structure described in Sec. IV B from 5 to $10 \mathrm{MeV}$. The attentive reader may remember from Sec. IVA that the ZTT advantage of IH cavities over the other solutions considered extended well beyond $10 \mathrm{MeV} / \mathrm{u}$. However, at the present stage the cost per unit power of $750 \mathrm{MHz}$ rf sources, currently only inductive output tubes (IOTs), is about 1 order of magnitude higher than the corresponding cost in the case of $3 \mathrm{GHz}$ klystron-modulator based solutions [29]. This difference is largely caused by the high demand of this latter technology working at $3 \mathrm{GHz}$, caused by the market of x-ray electron linacs. However, even though it is the authors' belief that this price difference will decrease in the future as a consequence of a higher demand of $750 \mathrm{MHz} r f$ power sources, at the present stage the crossing point between a $750 \mathrm{IH}$ solution and a $3 \mathrm{GHz}$ DTL one has been found at $10 \mathrm{MeV} / \mathrm{u}$.

To boost protons from 5 to $10 \mathrm{MeV}$ it has been chosen to use just one $100 \mathrm{~kW}$ IOT, resulting in a fairly low gradient of about $5.7 \mathrm{MV} / \mathrm{m}$, and in a $0.9 \mathrm{~m}$ long structure. The structure is tapered in length, and composed of $36 \mathrm{drift}$ tubes. The first cell is $20.8 \mathrm{~mm}$ long, the last one $28.9 \mathrm{~mm}$ long. The average ZTT, taking into consideration the end 
cells, is around $350 \mathrm{M} \Omega / \mathrm{m}$. The beam from the RFQ was transversally matched with a triplet quadrupole focusing, and then accelerated in the IH structure with a constant synchronous phase of $-12 \mathrm{deg}$. A $16 \%$ margin in the IOT nominal power was kept, to take into account waveguide losses, so $84 \mathrm{~kW}$ are actually dissipated by the cavity walls.

Given the small bore aperture and thin drift tubes, a not negligible dipole kick component is present, equal to almost $15 \%$ of the longitudinal component, as discussed in Sec. IV B 1. Indeed, when the beam was tracked through the structure field map with RF-TRACK, it showed a final displacement of about $0.7 \mathrm{~mm}$ out of a $2.5 \mathrm{~mm}$ bore aperture. This was clearly not acceptable, and it has been corrected in the following way.

In Fig. 24 (top) one can notice the $\mathrm{x}$ displacement of a single particle that enters the field map with no initial displacement and divergence. The particle gets deflected in the first gap, then drifts away in the drift tubes. In the second gap, the dipole kicks have opposite direction but almost equal magnitude. In fact, a IH cavity is a constant voltage structure, so also the transverse voltage is constant. This is true at first order approximation, given that the dipole kick component increases with longer cell lengths. However, so does the beam rigidity. Overall, the particle divergence gets approximately to zero in the second gap of the structure. Then in the third gap it picks again an $\mathrm{x}$ divergence, which gets canceled again in the fourth gap. So,

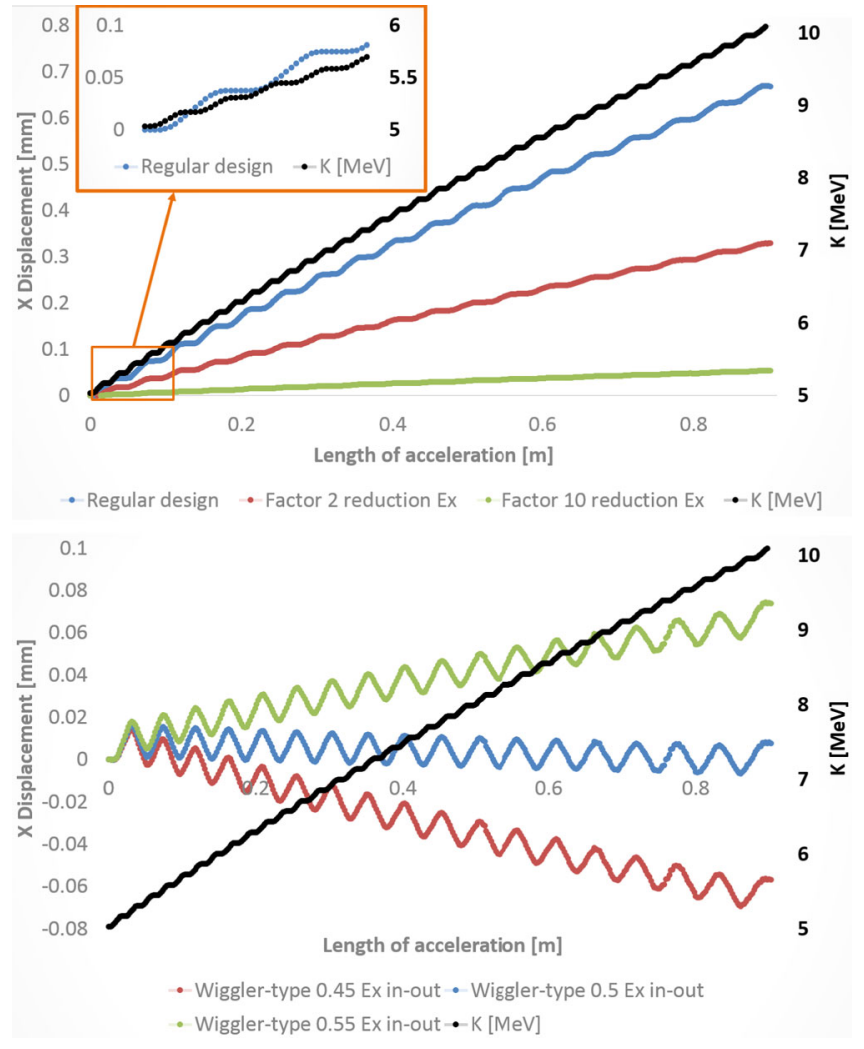

FIG. 24. Single particle tracking through the IH structure. Dipole kicks reduction (top) and undulator solution (bottom). traveling through cells, the particle shows a linear displacement with energy, and zero integrated kick. Solutions proposed in previous works [30] were based on the modification of the drift tube shape and relative position with respect to the $\mathrm{x}$ axis, to reduce the dipole component. For the IH cavity under study, this presents the disadvantage of reducing the ZTT with respect to the nominal solution. In addition, particles would still experience a linear displacement, simply a smaller one [Fig. 24 (top), red and green curve].

In this work a new solution is proposed. By halving the dipole kicks component in the first accelerating gap, also the divergence picked up by the particles is halved. As a consequence, the second gap, which has a "nominal" dipole kick, oversteers the beam, which has now a negative divergence. This solution is shown graphically in Fig. 24 (bottom), which shows the single particle displacement along the IH structure field map with this solution adopted. The trajectory of the particles recalls the one of an undulator. Eventually, the last gap must also have a half dipole kick component, to zero the integrated kick.

The beam was transversally matched using a triplet focusing [Fig. 25 (bottom)]. A relatively high synchronous phase of -12 deg was chosen only to facilitate the particles injection into the $3 \mathrm{GHz}$ DTL at $10 \mathrm{MeV}$. Indeed, a much lower synchronous phase (5 to $10 \mathrm{deg}$ ) would have been needed to accept and control the particle from the RFQ. The constant synchronous phase solution was adopted rather than the well-known KONUS [31] in view of its higher simplicity and robustness.

Concerning the longitudinal phase space, the Twiss $\alpha_{z}$ parameter of the RFQ beam was modified from 0 to 0.6 to improve the longitudinal matching. At the present stage, it has been assumed that this will be achievable with a different RFQ design [32]. Alternatively, one should consider to install a buncher cavity between the RFQ and the IH structure.

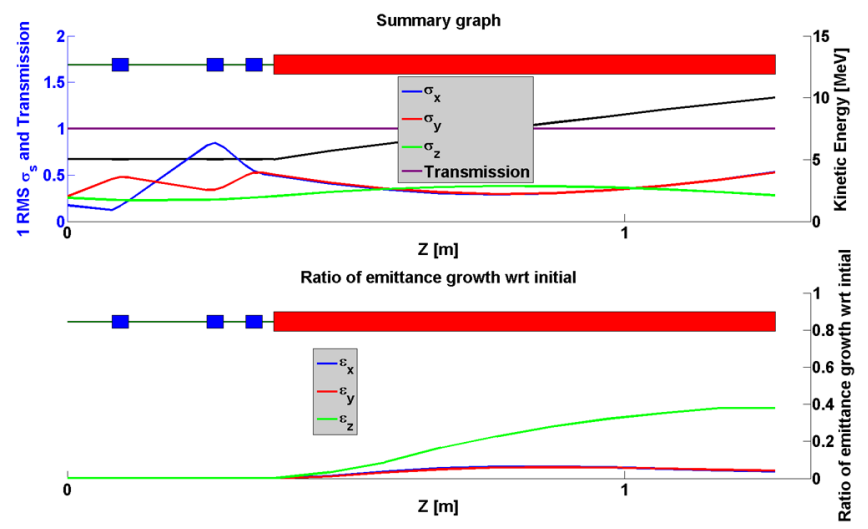

FIG. 25. Beam $1 \sigma$ rms envelope through the IH structure from 5 to $10 \mathrm{MeV}$ (top) and ratio of emittance growth with respect to initial (bottom). 
TABLE III. Beam dynamics comparison between a IH-DTL and a full DTL solution in the 5 to $20 \mathrm{MeV} / \mathrm{u}$ range.

\begin{tabular}{lcc}
\hline \hline Parameter & IH + DTL & DTL \\
\hline Synchronous phase $[\mathrm{deg}]$ & $-12(\mathrm{IH})$ and -20 (SCDTL) & $-30(5$ to $10 \mathrm{MeV})$ and $-25(10$ to $20 \mathrm{MeV})$ \\
Transmission & 100 & 99.6 \\
Transverse $\epsilon$ growth [\%] & 7 & 35 \\
Longitudinal $\epsilon$ growth [\%] & 53 & 166 \\
Total length [m] & 3.01 & 2.89 \\
Active length [m] & 1.68 & 1.26 \\
Number of accelerating structures & 10 & 19 \\
Number of PMQ & 15 & 20 \\
Peak power consumption $[\mathrm{MW}]$ & $0.1(750 \mathrm{MHz})+2.0(3 \mathrm{GHz})$ & $3.7(3 \mathrm{GHz})$ \\
\hline
\end{tabular}

\section{1. $750 \mathrm{MHz}$ IH vs $3 \mathrm{GHz}$ DTL beam dynamics comparison}

In Sec. IVA it was shown that up to $20 \mathrm{MeV} / \mathrm{u}$, the $750 \mathrm{MHz}$ IH solution is more efficient than a $3 \mathrm{GHz}$ DTL one from an rf efficiency point of view. In this section, the beam dynamics of the two solutions will be discussed. To get a fair comparison, here a IH (5 to $10 \mathrm{MeV}$ ) plus DTL (10 to $20 \mathrm{MeV}$ ) and full DTL (5 to $20 \mathrm{MeV}$ ) solutions will be compared. In such a way, it is possible to compare final beam parameters in similar phase space configurations.

The main aspects that have been compared are (i) particles transmission; (ii) emittance growth; (iii) overall length and space for diagnostic; (iv) number of elements; and (v) power consumption.

The authors decided to prioritize amongst all other parameters the beam transmission.

The SCDTL choice reveals to be particularly challenging in the 5 to $10 \mathrm{MeV}$ range. Together with machinability and tuning considerations, and not forgetting the rf efficiency already mentioned, the beam dynamics also represents an issue. The space between the RFQ and the first SCDTL module is limited by the longitudinal acceptance of the beam. This limits the transverse matching of the beam, with repercussion on the emittance growth. A solution could be the installation of a buncher cavity, which would allow a longer matching section. A second problem arises from the relatively high accelerating gradient. At $5 \mathrm{MeV}$, the ratio between active and total length is well below $50 \%$, since the rf defocusing has a square dependence with particle momentum [33]:

$$
\Delta p_{r}=-\frac{\pi e E_{0} T L r \sin \phi}{c \beta^{2} \gamma^{2} \lambda}
$$

and so it is necessary to have short accelerating tanks with PMQs in between. As a result, to accelerate over the same length, the gradient of the full DTL solution must be higher with respect to the IH-DTL solution. This results in a heavily longitudinally mismatched beam. Figure 28 shows the longitudinal phase space evolution of the beam in the first six DTL accelerating structures. As one can notice, the beam fits well in the first structure longitudinal bucket (red contour). However, the combination of high accelerating gradient and long drift sections between the different DTL tanks led to filamentation, eventually resulting in emittance growth and losses.

A higher synchronous phase would not help, since it will increase the rf defocusing. So shorter structures would be needed to transversally control the beam, and the accelerating gradient should be increased to keep the overall length constant. Two solutions could be followed. One could reduce the accelerating gradient to get a smoother acceleration in the first sections, but resulting in a longer linac. Alternatively, it would be possible to match the beam from tank to tank, by designing the DTL such that both the synchronous phase and the gradient adapt to the longitudinal orientation of the beam ellipse. This last proposal would raise significantly the rf design complexity of such linac.

The two designs are summarized in Table III and displayed graphically in Figs. 26 and 27. The overall dimensions are comparable in the two designs. However, the full DTL solution shows losses and a higher emittance growth. In addition, there is not space to allocate beam diagnostic. As a final comment, the full DTL solution requires more PMQs and accelerating structures, so a higher number of brazing and tuning procedures.

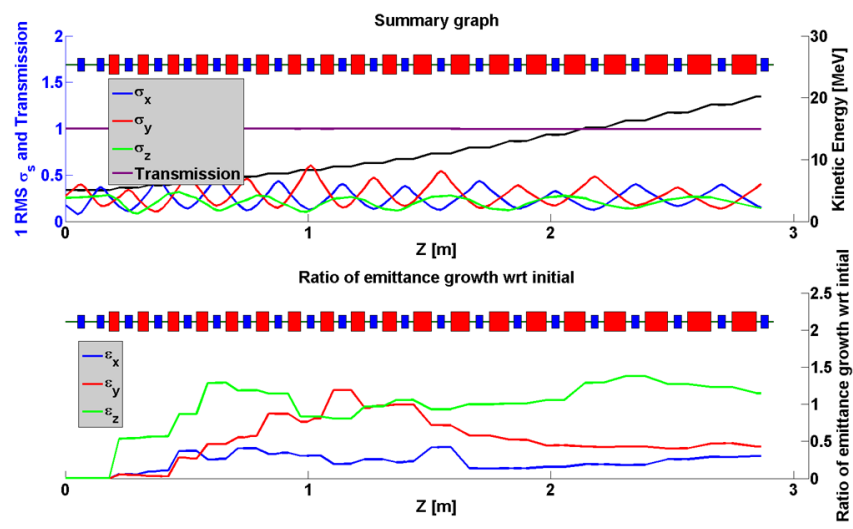

FIG. 26. Beam $1 \sigma$ rms envelope through a DTL structure from 5 to $20 \mathrm{MeV}$ (top) and ratio of emittance growth with respect to initial (bottom). 


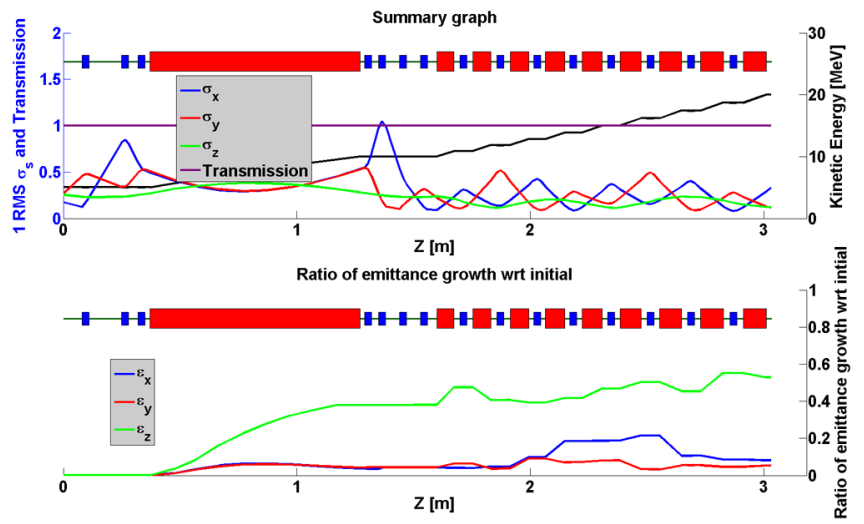

FIG. 27. Beam $1 \sigma \mathrm{rms}$ envelope through the $\mathrm{IH}+\mathrm{DTL}$ structure from 5 to $20 \mathrm{MeV}$ (top) and ratio of emittance growth with respect to initial (bottom).

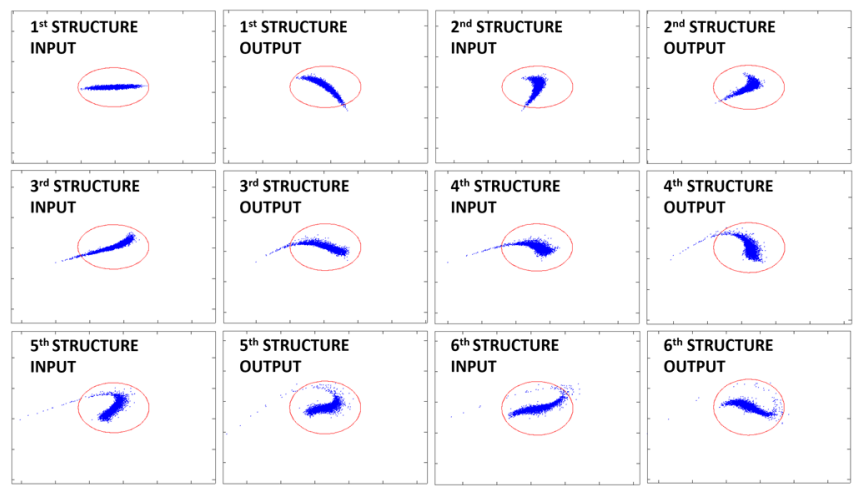

FIG. 28. Longitudinal phase space in and out the first six accelerating tanks of a DTL solution, from 5 to $7.7 \mathrm{MeV}$, with simplified buckets contour in red. The different pictures are in scale.

\section{From 10 to $70 \mathrm{MeV}$}

As it was demonstrated in Secs. IV B and VB 1, a IH-DTL solution is superior over a DTL from both an $\mathrm{rf}$ and a beam dynamics point of view. However, cost considerations led towards the decision of accelerating particles from $10 \mathrm{MeV}$ onwards with a $3 \mathrm{GHz}$ DTL linac.

The rf design has been discussed in Sec. IV C. Three modules tapered in length have been designed, each composed of nine accelerating tanks. The number of cells per tank goes from five in the first module, to six and seven in the second and last modules respectively. This in order to increase the ratio of active acceleration length over the total, taking advantage of the lower rf defocusing at higher particle momentum. The synchronous phase is constant and equal to $-20 \mathrm{deg}$ in all three modules. A solution with a higher number of cells in the first module was studied, but it was not feasible due to the too strong rf defocusing.

The main parameters of this linac section are summarized in Table IV.

The beam envelopes and emittance growth in this linac section are shown in Fig. 29.

\section{Matching between a $750 \mathrm{MHz}$ IH to a $3 \mathrm{GHz} \mathrm{DTL}$}

The beam matching between the $750 \mathrm{MHz} \mathrm{IH}$ and the $3 \mathrm{GHz}$ DTL is one of the most critical parts of the project. In the longitudinal phase space there is a reduction of a factor 4 in the phase acceptance, given by frequency increase. Concerning the energy acceptance, there is a factor 2 reduction, given by the square root dependence of $\lambda$, as shown in Eq. (8):

$$
\omega_{\max }=\sqrt{\frac{2 q E_{0} T \beta^{3} \gamma^{3} \lambda}{\pi m c^{2}}\left(\phi_{s} \cos \phi_{s}-\sin \phi_{s}\right)} .
$$

However a factor $\sqrt{\beta^{3} \gamma^{3}}$ helps in increasing the energy acceptance, which was one of the motivations in delaying the jump at $3 \mathrm{GHz}$ from 5 to $10 \mathrm{MeV} / \mathrm{u}$. In particular, going from 5 to $10 \mathrm{MeV}$, the acceptance for protons increases by a factor 0.22 .

In the transverse phase space, the geometric emittance decreases by a factor $\beta \gamma$, so by about $50 \%$ between 5 and $10 \mathrm{MeV}$. Nevertheless, while the RFQ is a bunching device, and it was specifically developed to inject particles into a $3 \mathrm{GHz}$ structure, the $\mathrm{IH}$ is an accelerating structure, so it was not obvious at the beginning to be capable of reaching a good transition and matching at $10 \mathrm{MeV}$.

A $33 \mathrm{~cm}$ long transverse matching section with four PMQs was designed to make the transition from a triplet focusing system, used for the IH structure, to a FODO lattice. The Twiss parameters were matched to have a phase advance of almost 90 deg in the SCDTL structures. A full transmission of the beam is reached, and the transverse emittance growth is well below 5\% at the end of the linac (Fig. 29). In the first linac sections there is a local transverse emittance growth up to $20 \%$, due to a residual initial mismatch. This effect cancels out due to the acceleration of the beam.

TABLE IV. SCDTL main parameters.

\begin{tabular}{lccccccc}
\hline \hline & $\begin{array}{c}\text { Output } \\
\text { Module }\end{array}$ & $\begin{array}{c}\text { Number } \\
\text { Energy [MeV] }\end{array}$ & $\begin{array}{c}\text { Active } \\
\text { of cells }\end{array}$ & $\begin{array}{c}\text { Ratio Active/Total } \\
\text { length }[\mathrm{m}]\end{array}$ & $\begin{array}{c}\text { Average active } \\
\text { Length }\end{array}$ & $\begin{array}{c}\text { Peak Power Average ZTT } \\
\text { Gradient }[\mathrm{MV} / \mathrm{m}]\end{array}$ & $\begin{array}{c}\text { AMW] } \\
{[\mathrm{M} \Omega / \mathrm{m}]}\end{array}$ \\
\hline 1 & 20 & 5 & 0.78 & 0.53 & 13.6 & 1.9 & 75 \\
2 & 40 & 6 & 1.31 & 0.64 & 16.2 & 3.7 & 94 \\
3 & 70 & 7 & 2.05 & 0.74 & 15.6 & 5.7 & 88 \\
\hline \hline
\end{tabular}




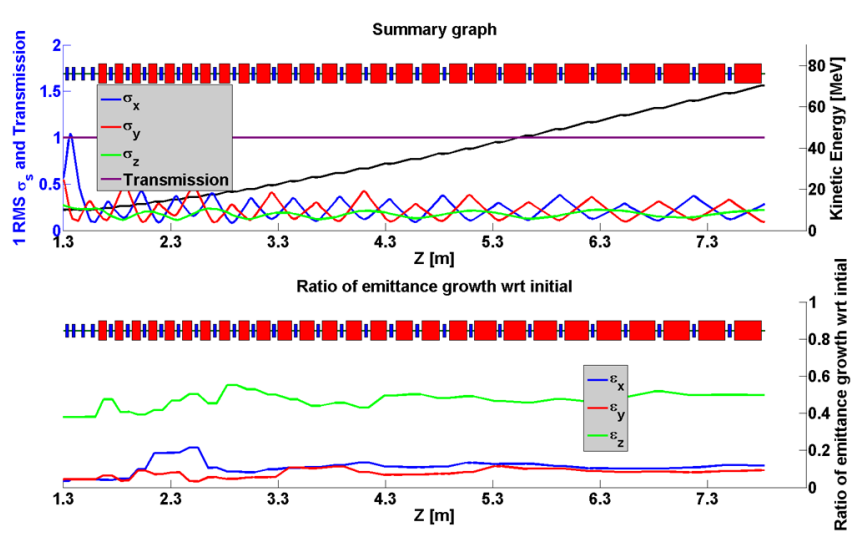

FIG. 29. Beam $1 \sigma \mathrm{rms}$ envelope through the DTL structure from 10 to $70 \mathrm{MeV}$ (top) and ratio of emittance growth with respect to initial (bottom).

\section{From 70 to $230 \mathrm{MeV}$}

The protons are eventually accelerated up to $230 \mathrm{MeV}$ in the high gradient linac. Following the considerations of Sec. IVE 1, the authors consider the BTW and the CCL solutions are ultimately even. However, we will review two designs, based on the two technologies, but comparable in length and number of elements. Before presenting the high gradient linac beam dynamics design, in the following section we review the theory of an energy varying beam line.

\section{Maximizing the energy acceptance of a beam line}

As the other 3 GHZ TERA linacs, the focusing is achieved by PMQs. The energy is varied by adjusting the powers and the phases of the rf pulses produced by the klystrons. In the case of protons, to reach the conventional range of penetration in water equivalent tissues, one needs protons ranging from 70 to $230 \mathrm{MeV}$.

In the following, we derive the condition to maximize the energy acceptance of a beam line composed of PMQs, for a given lattice geometry. One can start from the basic beam dynamics equations that can be found in the general particle accelerators books $[34,35]$.

The normalized transverse acceptance of a linac is given by

$$
A_{n}=\frac{\beta \gamma}{\beta^{+}} \cdot R_{\mathrm{bore}}^{2}
$$

For a given bore aperture, the linac transmission can be maximized by minimizing the Twiss betas. Also one can notice that the acceptance increases with the beam energy, because the geometric emittance shrinks. This is valid under the assumption that the normalized emittance remains constant.

It is convenient to express the Twiss beta in terms of phase advance. The FODO theory, even though not entirely correct when describing a linac, works well enough:

$$
\beta^{ \pm}=2 L \frac{1 \pm \sin (\mu / 2)}{\sin (\mu)}
$$

where $\mathrm{L}$ is the FODO length and $\mu$ is the transverse phase advance per cell.

In the case of a magnetic line with PMQs, the transverse phase advance and the Twiss $\beta_{s}$ seen by the beam vary with the output energy.

In a round beam, as the RFQ output, we have $\epsilon_{x} \approx \epsilon_{y}$, so the geometric dimension of the beam is given by $A=\pi \cdot R^{2}=\pi \cdot\left(\epsilon_{x} \beta_{x}+\epsilon_{y} \beta_{y}\right)$. The beam size is minimized in the case of phase advance of $90 \mathrm{deg}$, as one can verify by plugging Eq. (10) in the above expression, and take the derivative. Having however a varying phase advance as a function of the energy, one needs to find the best compromise that maximizes the acceptance in the range of energy of interest.

The optimum is found when the phase advance is $90 \mathrm{deg}$ at the minimum beam line energy, and then decreases as the beam energy increases. The decrease has to be such that

$$
\frac{\beta_{E_{\min }}^{+}}{\beta_{E_{\max }}^{+}}=\frac{(\beta \gamma)_{E_{\min }}}{(\beta \gamma) E_{\max }}
$$

which simply means that the beam envelope must have the same dimension in the two extreme cases at the end of the line. Since the $\beta^{+}$and the $\beta \gamma$ curves as a function of the final beam energy have a positive second order derivative, the worst conditions are found at the two extremes.

In conclusion, one shall follow these steps in the lattice design: (i) match transversally the beam for a 90 deg phase advance at the linac minimum energy section, in order to minimize the emittance growth; (ii) maximize the cumulative transmission at the minimum and maximum linac energy; the transmission in all other cases lies in between these two results; (iii) if there are losses, reduce the FODO length, or increase the beam aperture.

\section{The high gradient BTW linac}

An 18 accelerating structures linac was designed. The target gradient is $40 \mathrm{MV} / \mathrm{m}$, with maximum values of Sc and of the surface electric field equal to $0.48 \mathrm{MW} / \mathrm{mm}^{2}$ and $160 \mathrm{MV} / \mathrm{m}$, respectively. The linac is approximately $6.2 \mathrm{~m}$ long, and accelerates the particles up to $230 \mathrm{MeV}$. The gradient chosen does not fully exploit the limit at which the structure has been designed $(50 \mathrm{MV} / \mathrm{m})$ to maintain a safety margin in terms of BDR. In addition, the compactness goal of the structure was already reached with a $40 \mathrm{MV} / \mathrm{m}$ gradient. Finally, this $20 \%$ reduction in gradient translates into a $36 \%$ reduction in rf thermal load into the structure, allowing a higher DF of $0.01 \%$. The synchronous phase is $-15 \mathrm{deg}$ in all the accelerating structures. 


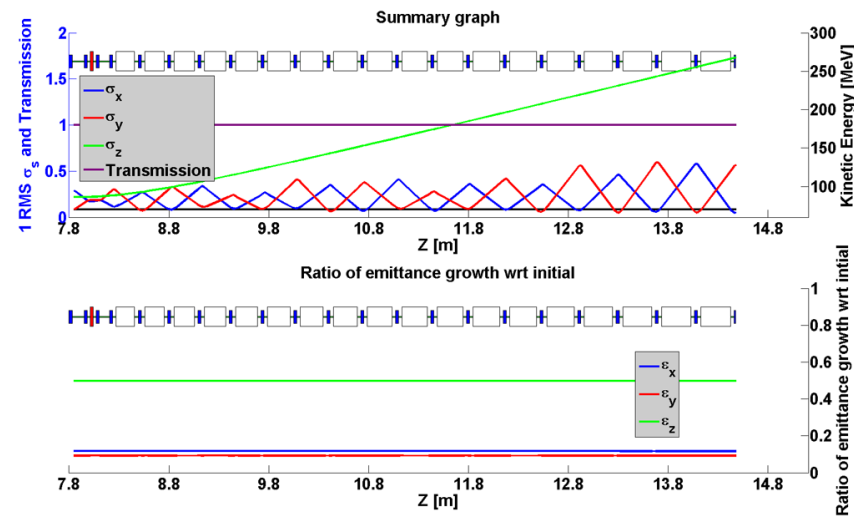

FIG. 30. Beam $1 \sigma$ rms envelope through the HG BTW linac with no acceleration after $70 \mathrm{MeV}$ (top) and ratio of emittance growth with respect to initial (bottom).

Varying the phase and amplitude of the rf power in each accelerating structure allows for a smooth variation of the final energy. For this reason, a single coupler solution has been studied (see Sec. IV E).

The emittances and beam envelopes are shown in Figs. 30 and 31 for the two extreme cases of no acceleration and full acceleration, respectively.

The matching section between the SCDTL and the BTW linac is considerably easier than the $10 \mathrm{MeV}$ transition. The solution proposed here comprises four PMQs and a buncher cavity to improve the longitudinal matching. This is just a temporary solution, given that the medium energy beam transfer line (MEBT), as shown in Fig. 2, is much longer and involves also dipoles. This part has not been studied yet since the mechanical design has yet to be finalized.

\section{The high gradient CCL linac and comparison with the BTW one}

The design presented above reaches full transmission of the particles between 70 and $230 \mathrm{MeV}$, but is however quite at the limit in terms of maximum Twiss beta. All the BTW accelerating structures are 12 cells long. This means that it is possible to obtain an exactly equivalent CCL design, composed of 18 accelerating structures, ten cells long.

As a result, the different between the two solutions translates in a different peak power consumption, accordingly to ZTT difference reported in Fig. 22. This difference is about $7 \%$ at $70 \mathrm{MeV}$ and gets null at $230 \mathrm{MeV}$.

\section{E. Beam dynamics in a cyclinac solution}

This article discusses the first design of an all-linac solution for the TULIP project. As mentioned, the majority of the studies carried out in the past focused instead on a cyclinac solution. Here a detailed beam dynamics study of this concept is presented, in order to point out the major differences.

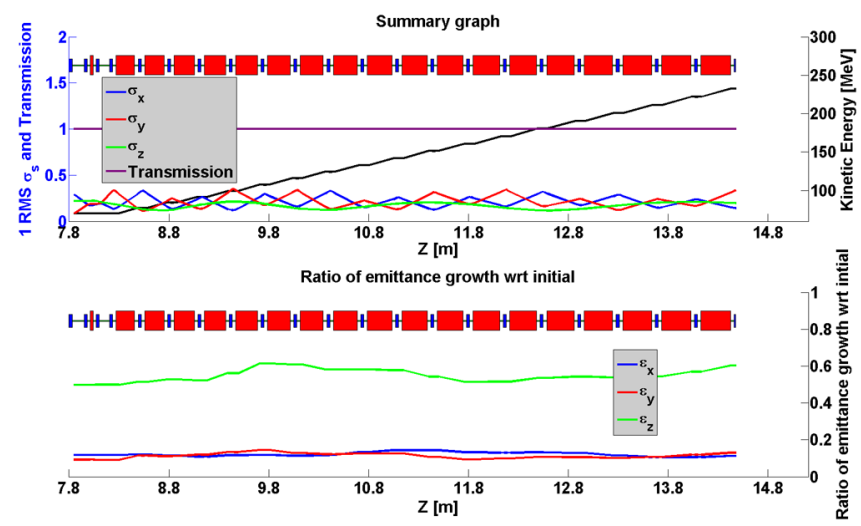

FIG. 31. Beam $1 \sigma \mathrm{rms}$ envelope through the HG BTW linac with full acceleration up to $230 \mathrm{MeV}$ (top) and ratio of emittance growth with respect to initial (bottom).

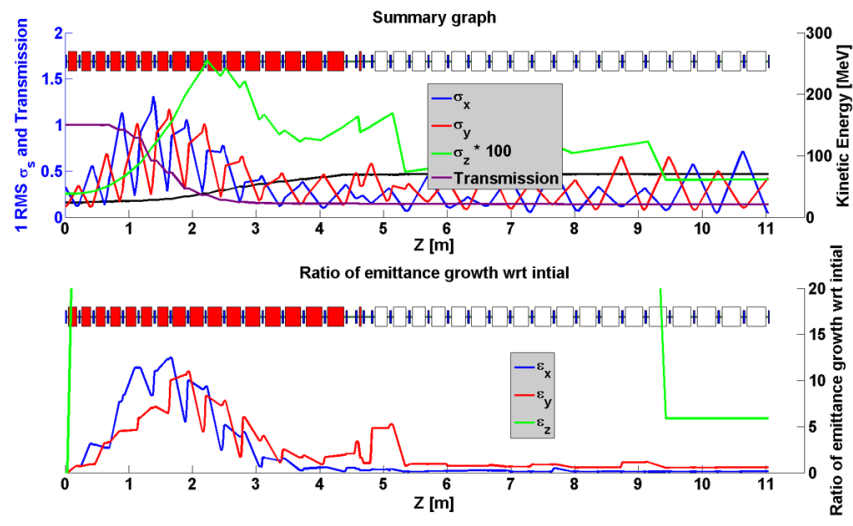

FIG. 32. Beam $1 \sigma$ rms envelope through a cyclinac TULIP solution with no acceleration after $70 \mathrm{MeV}$ (top) and ratio of emittance growth with respect to initial (bottom).

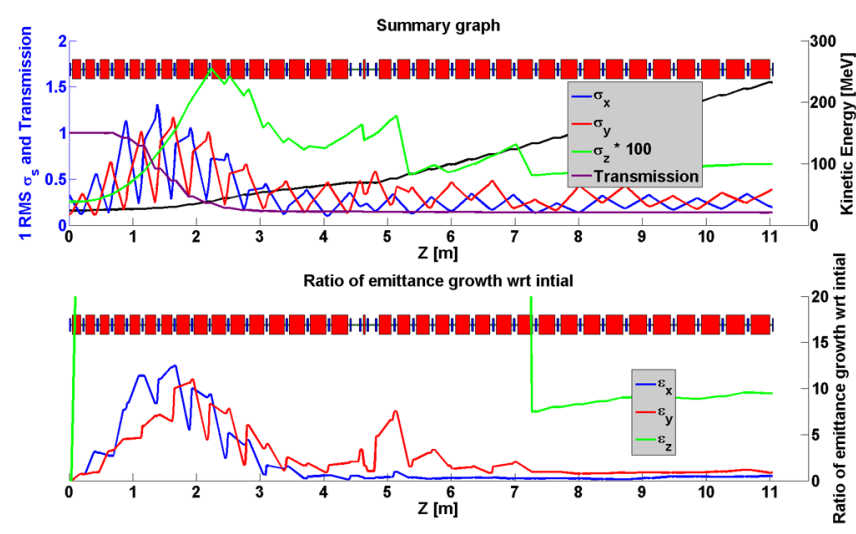

FIG. 33. Beam $1 \sigma$ rms envelope through a cyclinac TULIP solution with full acceleration up to $230 \mathrm{MeV}$ (top) and ratio of emittance growth with respect to initial (bottom). 


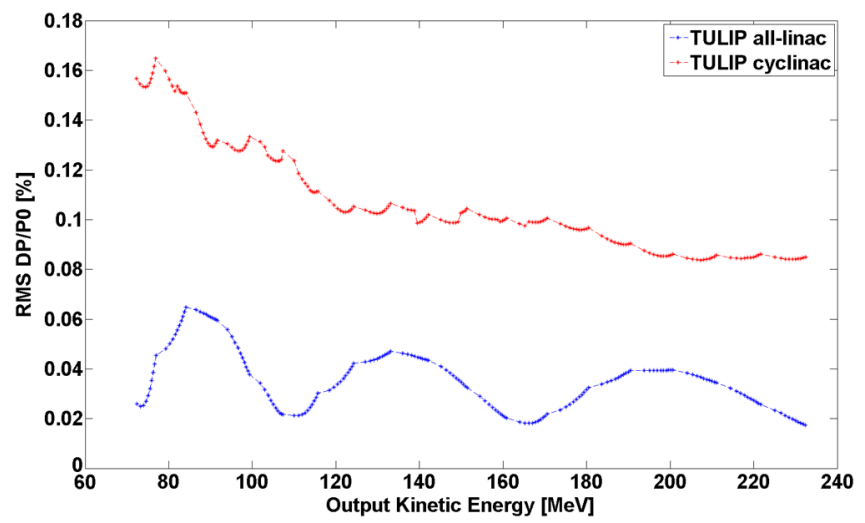

FIG. 34. Beam output energy spread in TULIP all-linac and cyclinac as a function of the final kinetic energy.

The 11th accelerating structure of the SCDTL linac accepts as input particles at $24 \mathrm{MeV}$. It has been supposed to replace the previous linac section with a commercial $24 \mathrm{MeV}$ cyclotron. This would result in a bigger transverse emittance, and a continuous beam for the $3 \mathrm{GHz}$ rf frequency, as discussed in Sec. VB 1. The transverse emittance can be collimated, however the beam cannot be chopped at $3 \mathrm{GHz}$. As a result, the beam will be lost in the linac section, with losses that are proportional to the ratio synchronous phase $-360 \mathrm{deg}$. This situation has been simulated, and the results are presented in Figs. 32 and 33.
The losses are concentrated in the first $3 \mathrm{~m}$ of this design, causing activation of the copper structure and surrounding materials. However, here we would like to draw the reader's attention to the emittance growth. While the transverse one is "controlled" by the linac acceptance, so it does not grow uncontrolled, the longitudinal phase space is heavily influenced.

These two aspects reflect into a higher complexity and costs of the beam transport line from the linac output to the patient. The increase in normalized transverse emittance requires bigger magnets aperture, therefore eventually costs. On the other hand, the increased longitudinal emittance results in a more variable energy spread as a function of the beam output energy. This impacts on the design of the magnetic channel, which needs a higher momentum acceptance to control the dispersion.

In Fig. 34 one can notice the difference just explained. The transverse Twiss parameters are instead comparable in magnitude for the two solutions.

\section{SUMMARY}

A all-linac solution of the TULIP project has been presented. In this paper we first discussed the rf design of the different accelerating structures composing the linac chain. Particular attention has been devoted to two novel accelerating structures studied in detailed by the authors: a $750 \mathrm{MHz} \mathrm{IH}$ structure, and a BTW high gradient structure. A $0.38 \beta$ BTW prototype has been
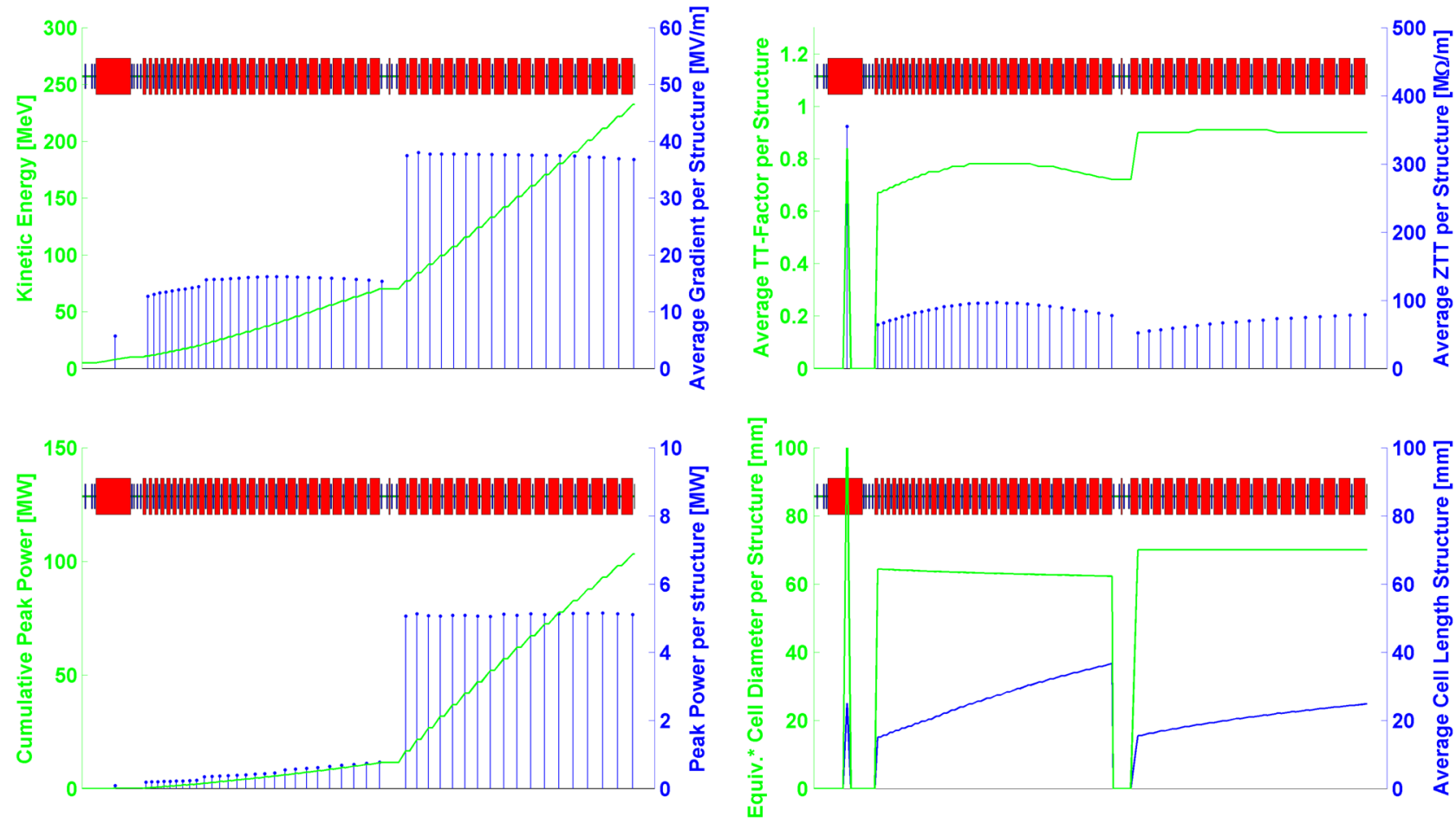

FIG. 35. TULIP all-linac main accelerating parameters. 
TABLE V. TULIP all-linac-A summary.

\begin{tabular}{lcccccccc}
\hline \hline $\begin{array}{l}\text { Linac } \\
\text { section }\end{array}$ & $\begin{array}{c}\text { Operating } \\
\text { frequency }[\mathrm{MHz}]\end{array}$ & $\begin{array}{c}\text { Output energy } \\
{[\mathrm{MeV}]}\end{array}$ & $\begin{array}{c}\text { Average gradient } \\
{[\mathrm{MV} / \mathrm{m}]}\end{array}$ & $\begin{array}{c}\text { Synchronous } \\
\text { phase }[\mathrm{deg}]\end{array}$ & $\begin{array}{c}\text { Active } \\
\text { length }[\mathrm{m}]\end{array}$ & $\begin{array}{c}\text { Cumulative } \\
\text { Length }[\mathrm{m}]\end{array}$ & $\begin{array}{c}\text { Average ZTT } \\
{[\mathrm{M} \Omega / \mathrm{m}]}\end{array}$ & $\begin{array}{c}\text { Peak power } \\
{[\mathrm{MW}]}\end{array}$ \\
\hline RFQ & 750 & 5 & 2.6 & 15 (final) & 2 & 2 & 38 & 0.4 \\
IH & 750 & 10 & 5.7 & 12 & 0.9 & 3.3 & 350 & 0.1 \\
DTL & 2998.5 & 70 & 15.5 & 20 & 4.1 & 9.8 & 86 & 13 \\
BTW-CCL & 2998.5 & $70-230$ & 37.7 & 15 & 4.4 & 17.5 & 68 & 108 \\
\hline \hline
\end{tabular}

built and is under test at CERN. The average target accelerating gradient in the structure is $50 \mathrm{MV} / \mathrm{m}$, with a corresponding maximum surface electric field of $200 \mathrm{MV} / \mathrm{m}$, and modified Poynting vector Sc of $0.75 \mathrm{MW} / \mathrm{mm}^{2}$.

The beam dynamics linac design features full transmission and minimized emittance growth, and it has been accomplished with full tracking of the particles from the RFQ output up to $230 \mathrm{MeV}$, using rf EM field maps for the accelerating structures computed with HFSS. This is a uniqum for such a long linac. The effort is justified by the peculiar cavities considered that, as discussed, show dipolar components, asymmetries and traveling wave regimes that
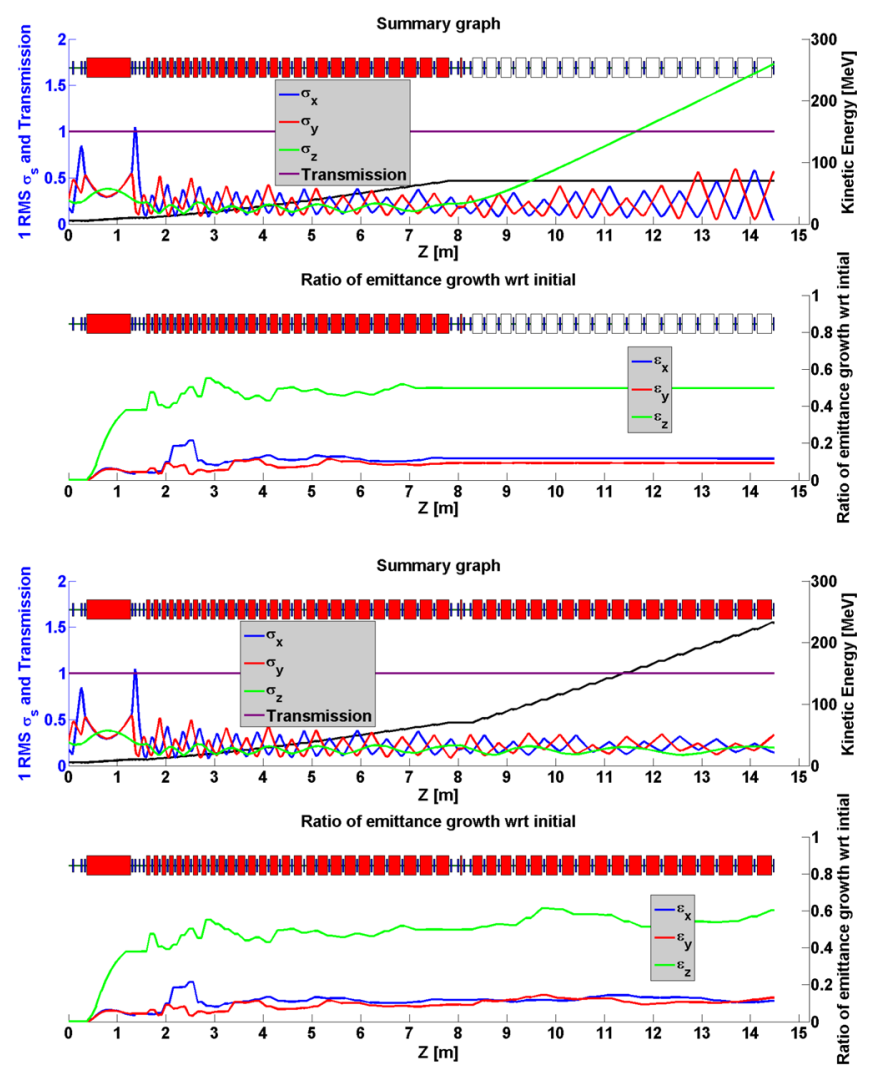

FIG. 36. Beam $1 \sigma$ rms envelope through the TULIP all-linac solution and ratio of emittance growth with respect to initial. No acceleration after $70 \mathrm{MeV}$ (top) and full acceleration up to $230 \mathrm{MeV}$ (bottom). cannot be simulated with standard tracking codes. This is the reason why the novel tracking code RF-TRACK has been developed explicitly for this project.

The TULIP all-linac solution with the main design parameters are summarized in Fig. 35 and Table V. Figure 35 top left and bottom left show clearly the division of the two sections of the project: a fairly low gradient and high efficiency section up to $70 \mathrm{MeV}$, placed on the ground, and a high gradient section that will be mounted on the gantry.

The beam dynamic design is summarized in Figs. 36 and 37 for the two extreme cases of no acceleration and full acceleration.
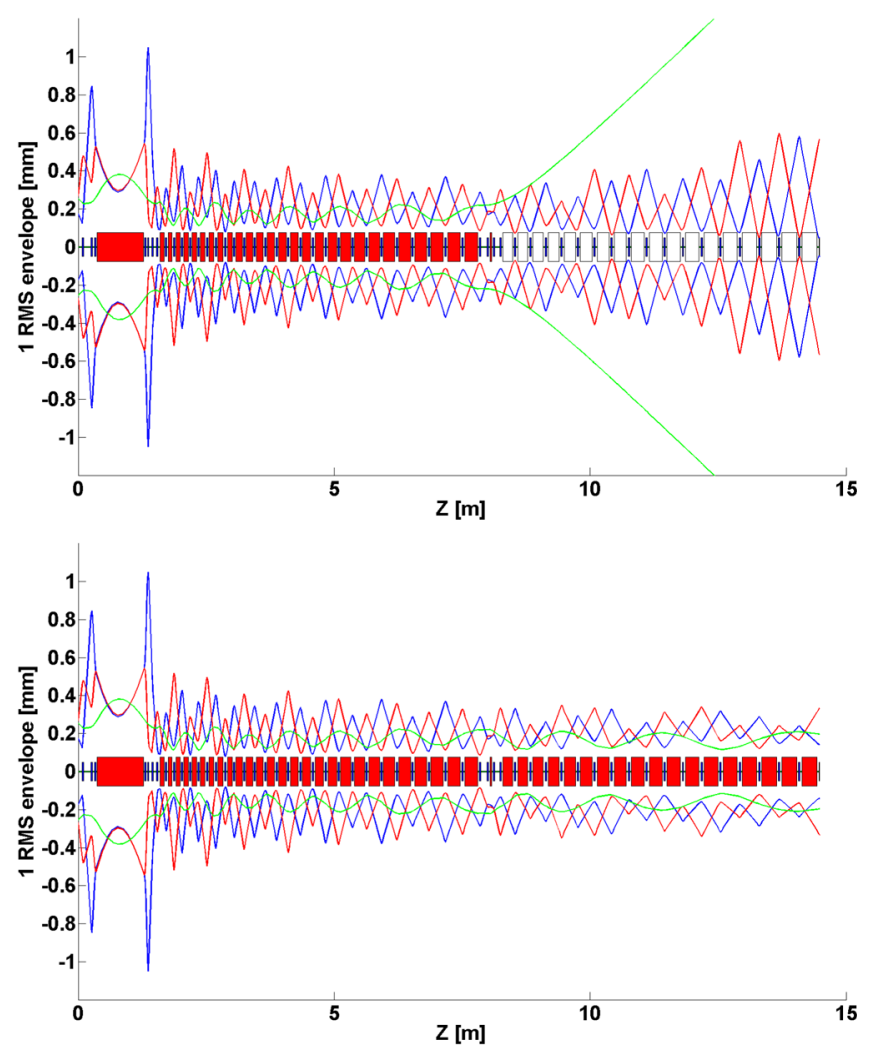

FIG. 37. The $70 \mathrm{MeV}$ (top) and $230 \mathrm{MeV}$ (bottom) beam envelopes along TULIP all-linac. $1 \mathrm{rms} \sigma_{x}$ (red), $\sigma_{y}$ (blue) and $\sigma_{z}$ (green). 


\section{ACKNOWLEDGMENTS}

The authors would like to sincerely thank Professor U. Amaldi for having initiated and supported this study. On different aspects, this work has relied on the fruitful contributions and discussions with many colleagues from TERA Foundation and CERN, in particular A. Degiovanni, M. Vaziri, M. Bucciantonio, I. Syratchev, W. Wuensch, E. Montesinos, A. Lombardi and V. Dimov.

[1] Particle Therapy Cooperative Group (PTCOG) Collaboration, http://www.ptcog.com.

[2] U. Amaldi, S. Braccini, and P. Puggioni, High frequency linacs for hadron therapy, Reviews of Accelerator Science and Technology (RAST) (World Scientific, Singapore, 2009), Vol. II, pp. 111-131.

[3] R. W. Hamm, K. R. Crandall, and J. M. Potter, Preliminary design of a dedicated proton therapy linac, in Proceedings of the 1991 Particle Accelerator Conference, San Francisco, CA, 1991 (IEEE, New York, 1991).

[4] U. Amaldi, M. Grandolfo, and L. Picardi, The RITA Network and the Design of Compact Proton Accelerators (INFN, Frascati, 1996), Chap. 9.

[5] U. Amaldi et al., LIBO-A linac booster for proton therapy: Construction and test of a prototype, Nucl. Instrum. Methods Phys. Res., Sect. A 521, 512 (2004).

[6] C. De Martinis et al., Acceleration tests of a $3 \mathrm{GHz}$ proton linear accelerator (LIBO) for hadron therapy, Nucl. Instrum. Methods Phys. Res., Sect. A 681, 10 (2012).

[7] C. Roncisvalle, L. Picardi, A. Ampollini, G. Bazzano, F. Marracino, P. Nenzi, C. Snels, V. Surrenti, M. Vadrucci, and F. Ambrosini, First acceleration of a proton beam in a side coupled drift tube linac, Europhys. Lett. 111, 14002 (2015).

[8] M. Vretenar et al., A compact high-frequency RFQ for medical applications, in Proceedings of 27th Linear Accelerator Conference, Geneva, Switzerland, 2014 (2014), pp. THPP040https://cds.cern.ch/record/2062619.

[9] http://www.advancedoncotherapy.com/Our-LIGHT-system/ Product-overview.

[10] A. Degiovanni et al., Design of a fast-cycling high-gradient rotating linac for proton therapy, in Proceedings of the 4th International Particle Accelerator Conference, IPAC-2013, Shanghai, China, 2013 (JACoW, Shanghai, China, 2013).

[11] A. Degiovanni et al., High gradient rf test results of S-band and C-band cavities (to be published).

[12] A. Degiovanni, High gradient proton linacs for medical applications, Ph.D. thesis, EPFL, 2014.

[13] A. Degiovanni et al., TERA high gradient test program of rf cavities for medical linear accelerators, Nucl. Instrum. Methods Phys. Res., Sect. A 657, 55 (2011).

[14] S. Benedetti et al., RF design of a novel S-band backward traveling wave linac for proton therapy, in Proceedings of 27th Linear Accelerator Conference, Geneva, Switzerland (2014), pp. THPP061http://cds.cern.ch/record/2062620.

[15] S. Benedetti et al., Fabrication and testing of a novel S-band backward traveling wave accelerating structure for proton therapy linacs, in Proceedings of the 28th Linear Accelerator Conference - LINAC16, East Lansing, MI, USA, 2016 (2016).

[16] P. Puggioni, Radio frequency design and measurements of a linear hadron accelerator for cancer therapy, M.Sci. thesis, Universita' degli studi Milano Bicocca, 2008.

[17] A. M. Lombardi, V. A. Dimov, M. Garlasche', A. Grudiev, S. Mathot, E. Montesinos, S. Myers, M. Timmins, and M. Vretenar, Beam dynamics in a high frequency RFQ, in Proceedings of the 6th International Particle Accelerator Conference (IPAC 2015): Richmond, Virginia, USA, 2015 (2015), pp. WEYB2, http://accelconf.web.cern.ch/Accel Conf/IPAC2015/papers/weyb2.pdf.

[18] U. Ratzinger, H-type linac structures. CERN Accelerator School: Radio frequency engineering, Seeheim, Germany, 2000.

[19] S. Benedetti, A. Grudiev, and A. Latina, Design of a $750 \mathrm{MHz}$ IH structure for medical applications, LINAC'16, East Lansing, MI, 2016.

[20] A. Grudiev, S. Calatroni, and W. Wuensch, New local field quantity describing the high gradient limit of accelerating structures, Phys. Rev. ST Accel. Beams 12, 102001 (2009).

[21] S. Verdu' Andre's, High-gradient accelerating structure studies and their application in hadron therapy, Ph.D. thesis, Universitat de Vale'ncia, 2012.

[22] I. Syratchev (private communication).

[23] S. Pitman, R. Apsimon, and G. Burt, ProBE: Proton boosting extension for imaging and therapy, in Proceedings of the 28th Linear Accelerator Conference LINAC16, East Lansing, MI, USA, 2016 (2016).

[24] A. Latina, RF-Track: Beam tracking in field maps including space-charge effects. Features and benchmarks, in Proceedings of the 28th Linear Accelerator Conference LINAC16, East Lansing, MI, USA, 2016 (2016).

[25] S. Benedetti et al., Design of a proton traveling wave linac with a novel tracking code, IPAC'15, Richmond, VA, 2015.

[26] http://www.desy.de/mpyflo/.

[27] S. B. van der Geer, General particle tracer: A 3D code for accelerator and beam line design, in Proceedings of the 6th European Particle Accelerator Conference, Stockholm, 1998 (IOP, London, 1998).

[28] E. Jensen, Superfish Exercise. Cern Accelerator Schoolrf for accelerators, Ebeltoft, Denmark, 2010 [arXiv: 1201.4648].

[29] E. Montesinos (private communication).

[30] S. Kurennoy et al., H-mode accelerating structures with permanent-magnet quadrupole beam focusing, Phys. Rev. ST Accel. Beams 15, 090101 (2012).

[31] R. Tiede, U. Ratzinger, H. Podlech, C. Zhang, and G. Clemente, KONUS beam dynamics designs using H-mode cavities, Hadron Beam 1, 2013 (2008).

[32] A. Lombardi and V. Dimov (private communication).

[33] M. Vretenar, Introduction to $\mathrm{rf}$ linear accelerators. Cern Accelerator School-General accelerator physics, Frascati, Italy, 2008.

[34] H. Wiedemann, Particle Accelerator Physics (Springer, New York, 2007).

[35] T.P. Wangler, $R F$ Linear Accelerators (Wiley-VCH, New York, 2008). 